\title{
Quantifying modern biomes based on surface pollen data in China
}

\author{
Yu Chen ${ }^{\mathrm{a}, \mathrm{b}}$, Jian $\mathrm{Ni}^{\mathrm{a}, \mathrm{c}, *}$, Ulrike Herzschuh $^{\mathrm{c}}$ \\ a State Key Laboratory of Vegetation and Environmental Change, Institute of Botany, Chinese Academy of Sciences, Xiangshan Nanxincun 20, 100093 Beijing, China \\ b Graduate University of Chinese Academy of Sciences, Yuquan Road Jia 19, 100049 Beijing, China \\ c Alfred Wegener Institute for Polar and Marine Research, Telegrafenberg A43, 14473 Potsdam, Germany
}

\section{A R T I C L E I N F O}

\section{Article history:}

Received 30 April 2010

Accepted 15 September 2010

Available online 13 October 2010

\section{Keywords:}

China

biome

biomization

human disturbance

model-data comparison

modern surface pollen

plant functional types

\begin{abstract}
A B S T R A C T
Large-scale surface pollen records and reconstructions of modern biomes are a necessary prerequisite for the understanding of past vegetation and climate changes, especially in large countries such as China which is subject to a variety of climatic regimes and has experienced long-term intensive anthropogenic disturbances. An updated surface pollen data set consisting of 2324 samples and 737 taxa is used to reconstruct biome distribution in China according to a newly established and well-tested global classification of plant functional types, based on the regional assessment of pollen taxa and the quantitative pollen-biome assignment method of biomization. Nineteen reconstructed types of biome present a reasonable reflection of the latitudinal and altitudinal distributions of modern vegetation in China. Incorrect assignment has previously occurred in some biomes, for example among the cold and cool temperate coniferous forests and mixed forest, among warmtemperate evergreen forest, mixed forest and tropical forests, and among temperate shrubland, grassland, desert and tundra biomes. Mega-biomes, grouped for the same bioclimatic zones, result in a better reconstruction than the nineteen separate biome types. The correct assignments increased from 68.8\% to $80.6 \%$. However, comparison of pollen-based biome reconstructions to climate-driven vegetation simulations performed using the global vegetation model BIOME4 indicates a low correlation rate (only 24.8\%), suggesting that more needs to be done to combine palaeoenvironmental data with model simulations of past vegetation changes. The misassignment of surface pollen to modern biomes usually occurs in areas which have similar bioclimatic features and vegetation types and for biomes which share the same plant functional types. These mis-matches often occur in mountainous regions where transitional vegetation zones occur on hill slopes at mid-altitudes. Our new modern biome reconstruction for China is more robust and reliable; however continued analysis of pollen records is required in the remote areas of western China and the Tibetan Plateau, as well as in regions of central and eastern China which have suffered from high levels of anthropogenic activity. This type of anthropogenic biome reconstruction presents a new challenge.
\end{abstract}

(c) 2010 Elsevier B.V. All rights reserved.

\section{Introduction}

Modern pollen assemblages, related to the current distribution of vegetation, land use and climate, provide a framework for inferring spatial and temporal variations in palaeovegetation and palaeoclimate from fossil pollen records (e.g. Overpeck et al., 1985; Prentice, 1985; Gajewski et al., 2002; Whitmore et al., 2005; Watrin et al., 2007). Modern pollen records, especially across broad regions, are therefore very useful for evaluating current pollen-vegetation relationships and for calibrating reconstructions of past vegetation and climate. In local and regional studies, modern pollen are closely related not only to vegetation and climate, but also to anthropogenically induced land use (e.g. Gaillard et al., 1994, 2008; Hjelle, 1999; Broström et al., 2004;

\footnotetext{
* Corresponding author. Alfred Wegener Institute for Polar and Marine Research, Telegrafenberg A43, D-14473 Potsdam, Germany. Tel.: +49 331288 2211; fax: +49 3312882137.

E-mail address: jni@awi.de (J. Ni).
}

Court-Picon et al., 2006). However, at extra-regional and continental scales, analysis of the distribution of modern pollen taxa is more focused on vegetation-pollen-climate relationships (e.g., Anderson et al., 1991; Gajewski et al., 2002; Whitmore et al., 2005; Watrin et al., 2007; Minckley et al., 2008), on pollen-vegetation relationships (e.g. Newsome, 1999; Markgraf et al., 2002; Ma et al., 2008) and on pollenclimate relationships (e.g. Seppä et al., 2004; Finsinger et al., 2007). These studies are all based on statistical approaches used to investigate pollen distributions and to reconstruct past changes in vegetation and climate. Less attention is paid to the impact of largescale land use change on modern pollen spectra (Liu et al., 2006, 2008).

The quantitative reconstruction of the large-scale geographical distribution of vegetation from pollen data using the concept of plant functional types (PFTs) is a commonly accepted method called 'biomization' (Prentice et al., 1996; Prentice and Webb, 1998). Continental and modern global biomes that can be used to compare and validate past vegetation changes during the mid-Holocene and at 
the last glacial maximum have been reconstructed from modern pollen records worldwide (see Prentice et al., 2000, and papers from two Special Features of Journal of Biogeography in 1998 and 2000, for the northern Hemisphere and Africa). Modern pollen-based largescale biome reconstructions have recently been extensively compared to natural vegetation in, for example, SE Asia, Australia and the Pacific (Pickett et al., 2004), Africa (Vincens et al., 2006; Lebamba et al., 2009), Latin America (Marchant et al., 2009) and Indian Continental (Sutra et al., unpublished). However these comparisons used potential modern biomes, i.e. by assigning vegetation types based on field observations and from various vegetation maps of natural biomes (all cultivated vegetation types were assigned to potential natural biomes in the same bioclimatic zones) in order to compare to the reconstructed biomes. Whilst modern pollen samples are normally taken from areas subject to minimal amounts of disturbance by human activity, this does not discount any influence from vegetation change and the impact of human activity in the past. Conversely, the biomization method can be used to reconstruct modern disturbed vegetation in highly disturbed regions such as Japan (Gotanda et al., 2008). This provides a method for investigating the impact of humans on vegetation during the late Holocene in Japan (Gotanda et al., 2008) and in other regions such as China, where there has been long-term human activity and historical land use changes.

Understanding the environmental history of China during the Quaternary has been of special interest to Earth System scientists. This is not only due to its large area, the diverse vegetation and broad climate regimes, but also due to the long history of human activities in China, including irrigated agriculture and forest clearance, in addition to the current environmental problems (Liu and Diamond, 2005). Addressing these problems requires a quantitative understanding of past environmental change and climate variability. A study of modern pollen, climate and vegetation is therefore a fundamental requirement for investigating these issues.

Studies of surface pollen at local and regional scales in China date back to the 1960s, but more comprehensive and quantitative research has only been conducted during the last decade. The Tibetan Plateau (Cour et al., 1999; Yu et al., 2001; Li et al., 2005; Shen et al., 2006, 2008; Herzschuh, 2007; Lu et al., 2008), the arid and semi-arid areas of northern and western China (Liu et al., 2006, 2008; Li et al., 2007; Xu et al., 2007, 2009), and areas of eastern China which have been highly disturbed by human activity (Zheng et al., 2007), are three major target regions for modern pollen studies. On a national level, the only studies which have been reported are those of arboreal pollen-vegetation relationships (Yu et al., 2004) and the biome reconstructions (Yu et al., 1998, 2000; Ni et al., 2010) using the biomization technique (Prentice et al., 1996, 2000; Prentice and Webb, 1998). Quantifying and reconstructing China's modern biomes from pollen data has been the basis for past biome reconstructions ( $\mathrm{Yu}$ et al., 2000; Ni et al., 2010). However, the limited number of pollen records and the uneven distribution of sampling sites in previous studies (the maximum is 806 samples only) has restricted the accurate interpolation of modern biomes to past vegetation and climate, as well as limited our understanding of biome boundaries. Disturbed modern vegetation types were treated as potential natural biomes, leading to inaccurate comparisons between reconstructed and observed modern biomes (Ni et al., 2010).

In this paper we use an extensive collection of new modern pollen records to quantitatively reconstruct modern biomes using the biomization method. We use a new global scheme of PFTs (Harrison et al., 2010, in preparation) that has been proven in China (Ni et al., 2010), and also conduct a more precise assignment of pollen taxa to PFTs based on newly published floras and online resources. The aims of this work are (1) to investigate the spatial variation of modern pollen-vegetation relationships in China, (2) to compare surface pollen-based biome reconstructions with modern vegetation including natural, potential and simulated biomes, and (3) to more precisely reconstruct modern biome distribution in order to better calibrate past vegetation changes.

\section{Data and methods}

\subsection{Modern pollen data}

The modern pollen data set comprises 2324 samples including 1770 raw pollen counts and 554 digitized ones (Fig. 1, Appendices A and B). Of them, 802 samples (764 raw and 38 digitized) were taken from previous publications of Chinese pollen biomization (Yu et al., 1998, 2000; Ni et al., 2010). Another 437 raw pollen samples were downloaded from the Eastern Asia Surface Pollen Dataset (http:// eapd.sysu.edu.cn/2/eapd.html). The remaining 569 raw samples were obtained from recent publications and unpublished resources. 516 digitized samples were hand-measured from pollen diagrams published largely between 2000 and 2008. Modern pollen samples were taken from a variety of deposition types, including soil surface (1589), moss polster (295), lake sediment surface (220), sediment core or profile tops (100), dust flux (80), marine sediment surface (19), and snow, ice and glacier (21). More detailed site information can be found in Appendix A.

The new expanded surface pollen data set has nearly three times the number of pollen samples than the previous one (Ni et al., 2010). Whilst geographical gaps in pollen sampling still exist, especially in the northern and northwestern desert areas, non-settlement areas of the Tibetan Plateau, mountainous regions of middle-southern China and some highly populated areas of eastern China, the current data set is the most updated version available. It comprises 181 more pollen taxa and 34 more taxon combinations compared to the previous data set.

\subsection{Biomization procedure}

The biomization procedure requires a list of pollen taxa, and the classifications of PFTs and biomes. In total we obtained 737 pollen taxa (species, genera and families) from the surface pollen dataset (Table 1). We cross-checked and standardized the different nomenclature used by various authors according to the Floras of China (ECFC, 1959-2002), and Dictionaries of Families and Genera of Chinese Seed Plants (Hou, 1998) and Ferns (Wu et al., 1992). The classification of PFTs in China adopted a newly established global scheme for PFTs (Harrison et al., 2010, in preparation) based on four principles: bioclimatic control (e.g. tropical, warm-temperate, temperate, boreal and arctic), phenology (e.g. evergreen or deciduous), leaf form (e.g. needle-leaved, broad-leaved, small-leaved and micro-leaved) and life form (e.g. tree, shrub, liana or vine, forb, climber, and fern). This scheme has been tested in a previous biomization of China based on limited surface pollen samples and fossil pollen records ( $\mathrm{Ni}$ et al., 2010). The eurythermic mesic and xeric drought-deciduous malacophyll low or high shrubs were modified to tropical ones because in China taxa from these two PFTs are restricted in tropical zones. Two additional PFTs, the warm-temperate drought-intolerant liana or vine, and climber were added. In total, 78 PFTs were used to establish the modern biomization of China (Table 1 ).

The recent biomization of China (Ni et al., 2010) used a total of 19 biomes, including 11 forest biomes, one shrubland, one grassland, one desert and five tundra biomes (Tables 2 and 3), and was based on the previous biome classifications of the world (Prentice et al., 1992; Kaplan, 2001; Kaplan et al., 2003) and of China (Yu et al., 1998, 2000). This type of biome scheme has its disadvantages, for example having overly complex biomes in cold- and cool-temperate zones and too few biomes in the warm-temperate (subtropical) zones (Ni et al., 2010). However, to date it is so far the best biome classification for palaeovegetation study in China and is comparable with the world biome system. Therefore we use this scheme in this study. Further 


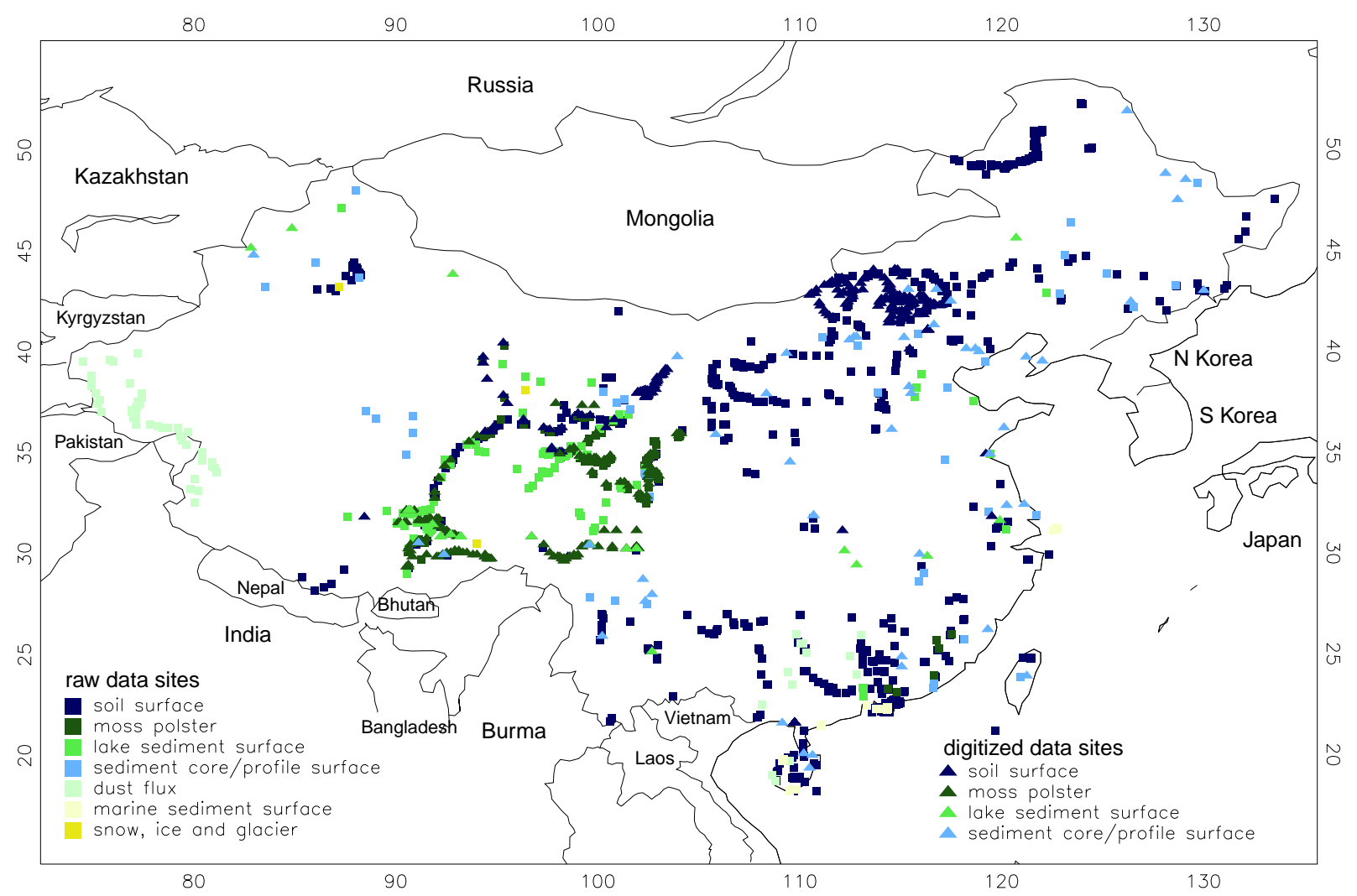

Fig. 1. Location of modern pollen samples.

details for this system are given in Table 2 based on the vegetation of China (ECVC, 1980) and the Vegetation Atlas of China (EBVAC, 2001).

We applied the standard biomization technique by following five steps (Prentice et al., 1996; Prentice and Webb, 1998):

(1) 737 pollen taxa were assigned to one or more of 78 PFTs (Table 1). The assignment was greatly improved in comparison to previous works (Yu et al., 1998, 2000; Ni et al., 2010) mostly due to the recent availability and correction of various floras and plant atlases (e.g. ECFC, 1959-2002; Wu et al., 1992; Hou, 1998; Fu et al., 1999-2009; Wu et al., 1994-2009). Of these, the assignments of 142 pollen taxa to PFTs were modified in relation to a change in PFT name, the update of various floras and the description of new PFTs mentioned previously. The improvement of such assignments include, for example, changing Ajania from "te-dt.fb" to "cs" and "rcf", adding "wt. cd.mb.t" to Fagus, adding "dt.sl.lhs" and "te-dt.fb" to Kochia, Malvaceae changing from "e" to "cd" in warm-temperate zones and to "dd" in tropical zones, adding "wt.e.mb.t" and "wt.e.mb. lhs" to Randia, and adding "tr.e.mb.t" to Sapindus (see Table 1 for more details). Some taxa were excluded from the biomization procedure and were not allocated to any PFTs as per Ni et al. (2010), including algae (e.g. Concentricystes, Mongeotia, Pediastrum, Spirogyra, Zygnema, Zygnemataceae, and Hystrichosphaera), fungi and indeterminate spores (e.g. monoletes and triletes).

(2) Key PFTs which characterize the composition and structure of certain biomes and determine their spatial distribution were used to define 19 biomes (Table 3). 25 PFTs such as the warmtemperate and eurythermic evergreen needle-leaved low and high shrubs, mangrove, most of liana or vines, climbers, forbs, and ferns, as well as the woody parasite, root parasite, hydrophyte, aquatic, geophyte, epiphyte, and moss, were not used to define biomes because of their relative unimportance in characterizing zonal vegetation (Table 3 ).

(3) The two previously discussed matrices, the pollen taxa vs. PFTs and PFTs vs. biomes, were multiplied to produce a pollen taxa vs. biomes matrix that directly links pollen taxa to biome types.

(4) The affinity scores for each biome were then calculated for all pollen samples using a summarizing equation $A_{i k}=$ $\sum_{j} \delta i j \sqrt{\left\{\max \left[0,\left(p_{j k}-\theta_{j}\right)\right]\right\}}$. Where $A_{i k}$ is the affinity of pollen sample $k$ for biome $i$; summation is over all taxa $j$; $\delta_{i j}$ is the entry ( 0 or 1 ) in the biome vs. taxon matrix for biome $i$ and taxon $j$; $p_{j k}$ are the pollen percentages; and $\theta_{j}$ is a threshold pollen percentage, here defined as $0.5 \%$, to reduce noise resulting from occasional pollen grains derived from long-distance transport or contamination. Taxon with low pollen percentage less than $0.5 \%$ was excluded from the affinity score calculation (for more details, see Prentice et al., 1996; Prentice and Webb, 1998).

(5) Finally the biome of each pollen sample with the highest affinity score was assigned, subject to a rule that the least PFTrich biome takes priority where the affinity scores for two or more biomes are equal.

The five-step biomization procedure was performed using Biomise 3 software (Ben Smith, Lund University). Four data files are prepared prior to running the software: a file containing pollen data with information on site-name, longitude, latitude and altitude; a nomenclature file which enables pollen taxa to be recognized and files of pollen taxa vs. PFTs and of PFTs vs. biomes.

\subsection{Modern vegetation data}

Information on modern vegetation distribution is required to compare with the reconstructed biome data. We relied on field observations (vegetation type and/or species compositions) recorded 
Table 1

The assignment of modern pollen taxa to plant functional types (PFTs). PFTs marked with $\left({ }^{*}\right)$ were not used in the final biomization procedure.

\begin{tabular}{lll}
\hline PFT code & PFT name & Boreal evergreen needle-leaved tree \\
\hline $\begin{array}{l}\text { bo.e.n.t } \\
\text { c-te.e.n.t }\end{array}$ & $\begin{array}{l}\text { Cool-temperate evergreen needle-leaved tree } \\
\text { te.e.n.t }\end{array}$ & Temperate evergreen needle-leaved tree \\
wt.e.n.t & Warm-temperate evergreen needle-leaved tree \\
eu.e.n.t & Eurythermic evergreen needle-leaved tree \\
bo.d.n.t & Boreal deciduous needle-leaved tree & \\
wt.d.n.t & Warm-temperate deciduous needle-leaved tree \\
wt.e.sb.t & Warm-temperate evergreen sclerophyll broad- \\
& leaved tree & \\
wt.e.mb.t & Warm-temperate evergreen malacophyll broad- & leaved tree
\end{tabular}

Pollen taxa

Abies, Juniperus, Picea, Pinus (Haploxylon), Pinus pumila, Sabina

Abies, Abies fargesii, Picea, Picea schrenkiana, Pinus koraiensis, Pinus pumila, Pinus sylvestris var. mongolica, Sabina, Taxus, Tsuga

No taxa

Cathaya, Cedrus, Cryptomeria, Cunninghamia, Cupressus, Dacrydium, Keteleeria, Podocarpus, Podocarpus macrophyllus, Sequoia, Taxodiaceae, Taxus, Tsuga Cupressaceae, Pinaceae, Pinus, Pinus (Diploxylon) Larix

Pseudolarix, Taxodiaceae, Taxodium

Aquifoliaceae, Buxaceae, Buxus, Castanopsis, Euphorbiaceae, Fagaceae, Flacourtia, Flacourtiaceae, Ilex, Lauraceae, Leguminosae, Lithocarpus, Myrtaceae, Olea, Oleaceae, Quercus, Quercus (evergreen), Rhamnaceae, Rutaceae, Ziziphus

Acacia, Acronychia, Adinandra, Alangiaceae, Altingia, Anacardiaceae, Apocynaceae, Aquifoliaceae, Araliaceae, Ardisia, Bignoniaceae, Bischofia, Boraginaceae, Bridelia, Camellia, Capparidaceae, Castanopsis, Celastraceae, Citrus, Clerodendrum, Clethra, Croton, Cyclobalanopsis, Dalbergia, Daphniphyllum, Dendropanax, Diospyros, Diplospora, Distylium, Ebenaceae, Elaeagnaceae, Elaeocarpaceae, Elaeocarpus, Euonymus, Euphorbiaceae, Eurya, Exbucklandia, Excoecaria, Fagaceae, Ficus, Garcinia, Gardenia, Glochidion, Gordonia, Guttiferae, Hamamelidaceae, Ilex, Illicium, Lagerstroemia, Lauraceae, Leguminosae, Ligustrum, Lithocarpus, Loropetalum, Lythraceae, Magnolia, Magnoliaceae, Mallotus, Manglietia, Melastomataceae, Michelia, Microtropis, Moraceae, Myrica, Myrica rubra, Myrsinaceae, Myrsine, Myrtaceae, Olacaceae, Olea, Oleaceae, Ormosia, Osmanthus, Pentaphylacaceae, Photinia, Phyllanthus, Pistacia, Prunus, Quercus, Quercus (evergreen), Quercus semicarpifolia, Randia, Rhamnaceae, Rhamnus, Rubiaceae, Rutaceae, Sabiaceae, Sapindaceae, Sapindus, Schefflera, Schima, Scrophulariaceae, Simaroubaceae, Solanaceae, Sterculiaceae, Styracaceae, Styrax, Sycopsis, Symplocaceae, Symplocos, Syzygium, Theaceae, Thymelaeaceae, Trochodendron, Verbenaceae, Wendlandia, Wikstroemia, Ziziphus

tr.e.sb.t Tropical evergreen sclerophyll broad-leaved tree Artocarpus, Caesalpinia, Canarium, Combretaceae, Elaeocarp

tr.e.mb.t Tropical evergreen malacophyll broad-leaved tree Annonaceae, Antidesma, Aphanamixis, Apocynaceae, Araliaceae, Artocarpus, Bignoniaceae, Bischofia Boraginaceae, Bridelia, Buxaceae, Canthium, Carallia, Casearia, Cassia, Celastraceae, Cephalomappa, Claoxylon, Combretaceae, Croton, Crypteronia, Decaspermum, Dendropanax, Dimocarpus confinis, Diplospora, Dodonaea, Elaeocarpaceae, Elaeocarpus, Endospermum, Epiprinus, Eucalyptus, Euphorbiaceae, Eurya, Exbucklandia, Ficus, Flacourtia, Flacourtiaceae, Garcinia, Gardenia, Gironniera, Glochidion, Gordonia, Guttiferae, Helicia, Heritiera, Heteropanax, Homalium, Homonoia, Hopea, Icacinaceae, Kleinhovia, Lauraceae, Leguminosae, Lithocarpus, Lythraceae, Macaranga, Malania, Mallotus, Melastomataceae, Microdesmis, Mimosaceae, Moraceae, Myristicaceae, Myrsinaceae, Myrtaceae, Myrtus, Neonauclea, Nephelium, Nyctaginaceae, Olacaceae, Olea, Oleaceae, Ormosia, Pandanaceae, Pandanus tectorius, Papilionaceae, Pentaphylacaceae, Phyllanthus, Platea, Proteaceae, Pterolobium, Randia, Randia merrillii, Rhodoleia, Rhodomyrtus, Rubiaceae, Rutaceae, Sabiaceae, Sapindaceae, Sapindus, Sapotaceae, Schefflera, Schoepfia, Scrophulariaceae, Simaroubaceae, Sterculia, Sterculiaceae, Styracaceae, Syzygium, Tamarindus, Terminalia Thymelaeaceae, Tiliaceae, Ulmaceae, Ulmus, Vatica, Verbenaceae, Vitex, Wendlandia

bo.cd.mb.t Boreal cold-deciduous malacophyll broad-leaved Alnus, Betula, Betula albo-sinensis, Betula costata, Betula dahurica, Betula middendorfii, Betula platyphylla, tree $\quad$ Betulaceae, Populus, Salicaceae, Salix

te-ft.cd.mb.t Temperate (spring-frost tolerant) cold-deciduous Acer, Aceraceae, Alnus, Betula, Betula albo-sinensis, Betula dahurica, Betula middendorfii, Betula platyphylla, malacophyll broad-leaved tree Betulaceae, Corylus, Fagaceae, Fraxinus, Fraxinus mandshurica, Leguminosae, Papilionaceae, Populus, Quercus, Quercus (deciduous), Rosaceae, Salicaceae, Salix, Tilia, Tiliaceae, Toona sinensis, Ulmaceae, Ulmus Aesculus, Ailanthus, Anacardiaceae, Aralia, Araliaceae, Carpinus, Celastraceae, Celtis, Cornaceae, Cornus, Cotinus, Crataegus, Diospyros, Ebenaceae, Euphorbiaceae, Fagaceae, Ginkgo, Gleditsia, Guttiferae, Leguminosae, Magnolia, Magnoliaceae, Morus, Oleaceae, Papilionaceae, Platanus, Prunus, Quercus, Quercus (deciduous), Rhamnaceae, Rhamnus, Rhus, Rosaceae, Rutaceae, Salicaceae, Salix, Sambucus, Scrophulariaceae, Sorbus, Syringa, Toxicodendron, Ulmaceae, Ulmus, Verbenaceae, Vitex, Ziziphus Acer, Aceraceae, Bignoniaceae, Broussonetia, Caprifoliaceae, Carpinus, Carya, Castanea, Celtis, Elaeagnaceae, Eucommiaceae, Euonymus, Euptelea, Fagaceae, Fagus, Fraxinus, Ginkgo, Hamamelidaceae, Idesia, Juglandaceae, Juglans, Koelreuteria, Lagerstroemia, Leguminosae, Liquidambar, Liquidambar formosana, Lythraceae, Melia, Meliaceae, Meliosma, Moraceae, Morus, Ostrya, Papilionaceae, Phyllanthus, Pistacia, Platycarya, Platycarya strobilacea, Populus, Pterocarya, Pteroceltis, Quercus, Quercus (deciduous), Rosaceae, Sabiaceae, Salicaceae, Salix, Sambucus, Simaroubaceae, Tilia, Tiliaceae, Ulmaceae, Zelkova Aceraceae, Adina, Aesculus, Aesculus wilsonii, Ailanthus, Albizia, Alchornea, Alnus, Anacardiaceae, Araliaceae, Betulaceae, Boraginaceae, Broussonetia, Caprifoliaceae, Carya, Celtis, Cornaceae, Cornus, Corylopsis, Crataegus, Cyclocarya, Engelhardtia, Eucommiaceae, Euphorbiaceae, Euptelea, Fagus, Flacourtiaceae, Gleditsia, Hamamelidaceae, Hamamelis, Idesia, Juglandaceae, Juglans, Koelreuteria, Lagerstroemia, Lauraceae, Leguminosae, Liquidambar, Liriodendron, Lythraceae, Magnolia, Magnoliaceae, Malvaceae, Melia, Meliaceae, Meliosma, Mimosa, Mimosaceae, Moraceae, Morus, Myrica, Nyssa, Phyllanthus, Pistacia, Platanus, Platycarya, Platycarya strobilacea, Prunus, Pterocarya, Pteroceltis, Rhamnaceae, Rhamnus, Rhoiptelea, Rhus, Rosaceae, Sambucus, Sapium, Schoepfia, Simaroubaceae, Sorbus, Sterculiaceae, Styracaceae, Styrax, Thymelaeaceae, Toxicodendron, Toxicodendron succedaneum, Urticaceae, Verbenaceae, Vitex, Wikstroemia, Zelkova, Ziziphus Albizia, Anacardiaceae, Bombacaceae, Caesalpinia, Celastraceae, Chukrasia, Combretaceae, Engelhardtia, Euphorbiaceae, Flacourtiaceae, Hainania, Lannea, Leguminosae, Malvaceae, Meliaceae, Microcos, Mimosa, Mimosaceae, Oleaceae, Papilionaceae, Rubiaceae, Sapindaceae, Sapium, Spondias, Styracaceae, Tiliaceae, Trema, Ulmaceae, Ulmus Acacia, Bombacaceae, Capparidaceae, Cassia, Dalbergia, Dodonaea, Euphorbiaceae, Flacourtia, Flacourtiaceae, Leguminosae, Mimosaceae, Papilionaceae, Randia, Rubiaceae, Terminalia, Tiliaceae, Wendlandia, Ziziphus Casuarina

Elaeagnaceae, Elaeagnus, Leguminosae, Mimosa, Mimosaceae, Papilionaceae, Tamaricaceae, Tamarix, Ziziphus, Zygophyllaceae, Zygophyllum 
Table 1 (continued)

\begin{tabular}{|c|c|c|}
\hline PFT code & PFT name & Pollen taxa \\
\hline tu.t & Tuft tree & Areca, Cocos, Cycas, Palmae, Phoenix, Trachycarpus \\
\hline ar.e.n.lhs & $\begin{array}{l}\text { Arctic evergreen needle-leaved low and high } \\
\text { shrub }\end{array}$ & Pinus pumila \\
\hline wt.e.n.lhs* & $\begin{array}{l}\text { Warm-temperate evergreen needle-leaved low } \\
\text { and high shrub }\end{array}$ & Dacrydium, Podocarpus, Taxus \\
\hline eu.e.n.lhs* & $\begin{array}{l}\text { Eurythermic evergreen needle-leaved low and } \\
\text { high shrub }\end{array}$ & Cupressaceae, Pinaceae \\
\hline ft.ml.lhs & Frost-tolerant micro-leaved low and high shrub & Ephedra, Ephedra distachya, Ephedra equisetina, Ephedraceae, Myricaria, Reaumuria, Tamaricaceae, Tamarix \\
\hline dt.sl.Ihs & $\begin{array}{l}\text { Drought-tolerant small-leaved low and high } \\
\text { shrub }\end{array}$ & $\begin{array}{l}\text { Alhagi, Calligonum, Caragana, Ceratoides, Chenopodiaceae, Chenopodium, Elaeagnaceae, Elaeagnus, } \\
\text { Euphorbia, Euphorbiaceae, Hippophae, Kochia, Leguminosae, Mimosaceae, Nitraria, Papilionaceae, Potaninia, } \\
\text { Reaumuria, Salsola, Solanaceae, Tetraena, Thymus, Zygophyllaceae, Zygophyllum }\end{array}$ \\
\hline di.sl.lhs & $\begin{array}{l}\text { Drought-intolerant small-leaved low and high } \\
\text { shrub }\end{array}$ & Empetrum, Ericaceae \\
\hline wt.e.sb.lhs & $\begin{array}{l}\text { Warm-temperate evergreen sclerophyll broad- } \\
\text { leaved low and high shrub }\end{array}$ & $\begin{array}{l}\text { Aquifoliaceae, Buxaceae, Buxus, Euphorbiaceae, Fagaceae, Flacourtia, Flacourtiaceae, Ilex, Olea, Oleaceae, } \\
\text { Quercus, Quercus (evergreen), Rhamnaceae, Ziziphus }\end{array}$ \\
\hline ar.e.mb.lhs & $\begin{array}{l}\text { Arctic evergreen malacophyll broad-leaved low } \\
\text { and high shrub }\end{array}$ & Empetrum, Ledum, Ledum palustre \\
\hline bo.e.mb.lhs & $\begin{array}{l}\text { Boreal evergreen malacophyll broad-leaved low } \\
\text { and high shrub }\end{array}$ & Ericaceae, Ledum, Ledum palustre, Oxycoccus, Rhododendron, Ribes, Vaccinium, Vaccinium uliginosum \\
\hline wt.e.mb.lhs & $\begin{array}{l}\text { Warm-temperate evergreen malacophyll broad- } \\
\text { leaved low and high shrub }\end{array}$ & $\begin{array}{l}\text { Acacia, Adinandra, Anacardiaceae, Apocynaceae, Aporusa, Aquifoliaceae, Araliaceae, Ardisia, Asclepiadaceae, } \\
\text { Berberidaceae, Bignoniaceae, Boraginaceae, Bridelia, Cajanus, Camellia, Capparidaceae, Capparis, } \\
\text { Caprifoliaceae, Castanopsis, Celastraceae, Citrus, Clerodendrum, Clethra, Cornaceae, Croton, Damnacanthus, } \\
\text { Daphne, Daphniphyllum, Dendropanax, Diospyros, Diplospora, Distylium, Ebenaceae, Elaeagnaceae, Elaeagnus, } \\
\text { Elaeocarpaceae, Ericaceae, Euonymus, Euphorbiaceae, Eurya, Excoecaria, Fagaceae, Ficus, Garcinia, Gardenia, } \\
\text { Glochidion, Guttiferae, Hamamelidaceae, Ilex, Illicium, Itea, Jasminum, Lauraceae, Leguminosae, Ligustrum, } \\
\text { Lonicera, Loropetalum, Lycium, Lythraceae, Maesa, Magnoliaceae, Mallotus, Melastomataceae, Michelia, } \\
\text { Microtropis, Moraceae, Myrica, Myrsinaceae, Myrsine, Myrtaceae, Nandina, Nerium, Olacaceae, Olea, } \\
\text { Oleaceae, Osmanthus, Photinia, Phyllanthus, Pistacia, Prunus, Pterolobium, Quercus, Quercus (evergreen), } \\
\text { Randia, Rhamnaceae, Rhammus, Rhaphiolepis, Rhododendron, Rhododendron fargesii, Rubiaceae, Rutaceae, } \\
\text { Sabiaceae, Sapindaceae, Schefflera, Scrophulariaceae, Simaroubaceae, Skimmia, Solanaceae, Sterculiaceae, } \\
\text { Styracaceae, Styrax, Sycopsis, Symplocaceae, Symplocos, Syzygium, Theaceae, Thymelaeaceae, } \\
\text { Trochodendron, Vaccinium, Verbenaceae, Viburnum, Wendlandia, Wikstroemia, Zanthoxylum, Ziziphus }\end{array}$ \\
\hline tr.e.mb.lhs & $\begin{array}{l}\text { Tropical evergreen malacophyll broad-leaved low } \\
\text { and high shrub }\end{array}$ & $\begin{array}{l}\text { Acacia, Adinandra, Aglaia, Allomorphia, Allophylus, Annonaceae, Antidesma, Aphanamixis, Apocynaceae, } \\
\text { Aporusa, Araliaceae, Asclepiadaceae, Bignoniaceae, Boraginaceae, Bridelia, Buxaceae, Canthium, Casearia, } \\
\text { Cassia, Celastraceae, Claoxylon, Combretaceae, Croton, Decaspermum, Dendropanax, Diplospora, Dodonaea, } \\
\text { Elaeocarpaceae, Epiprinus, Eucalyptus, Eurya, Ficus, Flacourtiaceae, Garcinia, Gardenia, Gironniera, Glochidion, } \\
\text { Guttiferae, Hedyotis, Helicteres, Heteropanax, Homalium, Homonoia, Icacinaceae, Lardizabalaceae, Lauraceae, } \\
\text { Leguminosae, Lythraceae, Macaranga, Mallotus, Melastomataceae, Microdesmis, Mimosaceae, Moraceae, } \\
\text { Morinda, Myristicaceae, Myrsinaceae, Myrtaceae, Myrtus, Neonauclea, Nerium, Nyctaginaceae, Olea, } \\
\text { Oleaceae, Pandanaceae, Papilionaceae, Phyllanthus, Piper, Piperaceae, Proteaceae, Pterolobium, Randia, } \\
\text { Rhaphiolepis, Rhodoleia, Rhodomyrtus, Ricinus, Rubiaceae, Sabiaceae, Sapindaceae, Sapotaceae, Sauropus, } \\
\text { Schizomussaenda, Schoepfia, Scrophulariaceae, Simaroubaceae, Skimmia, Sterculiaceae, Styracaceae, } \\
\text { Syzygium, Verbenaceae, Vitex, Wendlandia }\end{array}$ \\
\hline ar.cd.mb.lhs & $\begin{array}{l}\text { Arctic cold-deciduous malacophyll broad-leaved } \\
\text { low and high shrub }\end{array}$ & Hydrangea, Oxytropis, Spiraea, Viburnum \\
\hline bo.cd.mb.lhs & $\begin{array}{l}\text { Boreal cold-deciduous malacophyll broad-leaved } \\
\text { low and high shrub }\end{array}$ & $\begin{array}{l}\text { Alnus, Betula, Betulaceae, Ericaceae, Guttiferae, Hypericum, Oxytropis, Rhododendron, Ribes, Salicaceae, Salix, } \\
\text { Viburnum }\end{array}$ \\
\hline te.cd.mb.lhs & $\begin{array}{l}\text { Temperate cold-deciduous malacophyll broad- } \\
\text { leaved low and high shrub }\end{array}$ & $\begin{array}{l}\text { Abelia, Acalypha, Acer, Aceraceae, Alnus, Anacardiaceae, Aralia, Araliaceae, Asclepiadaceae, Berberidaceae, } \\
\text { Berberis, Betula, Betulaceae, Bignoniaceae, Broussonetia, Caprifoliaceae, Caragana, Celastraceae, Celtis, } \\
\text { Cornaceae, Cornus, Corylus, Cotinus, Cotoneaster, Crataegus, Daphne, Diospyros, Ebenaceae, Elaeagnaceae, } \\
\text { Elaeagnus, Ericaceae, Euonymus, Euphorbiaceae, Euptelea, Fagaceae, Fontanesia, Gleditsia, Glochidion, } \\
\text { Guttiferae, Hamamelidaceae, Helwingia, Hippophae, Hydrangea, Hypericum, Ilex, Koelreuteria, Labiatae, } \\
\text { Lagerstroemia, Leguminosae, Lespedeza, Ligustrum, Lonicera, Lycium, Lythraceae, Malvaceae, Melia, } \\
\text { Meliaceae, Meliosma, Moraceae, Morus, Oleaceae, Ostryopsis, Oxytropis, Papilionaceae, Phyllanthus, Pistacia, } \\
\text { Prunus, Quercus, Quercus (deciduous), Rhamnaceae, Rhamnus, Rhododendron, Rhus, Ribes, Rosa, Rosaceae, } \\
\text { Rubus, Rutaceae, Sabiaceae, Salicaceae, Salix, Sambucus, Scrophulariaceae, Solanaceae, Sorbaria, Sorbus, } \\
\text { Spiraea, Syringa, Tiliaceae, Toxicodendron, Ulmaceae, Vaccinium, Verbenaceae, Viburnum, Violaceae, Vitex, } \\
\text { Vitex negundo var.heterophylla, Weigela, Wikstroemia, Zanthoxylum, Zelkova, Ziziphus }\end{array}$ \\
\hline wt.cd.mb.lhs & $\begin{array}{l}\text { Warm-temperate cold-deciduous malacophyll } \\
\text { broad-leaved low and high shrub }\end{array}$ & $\begin{array}{l}\text { Abelia, Acalypha, Acanthaceae, Adina, Albizia, Alchornea, Anacardiaceae, Aralia, Araliaceae, Asclepiadaceae, } \\
\text { Berberidaceae, Berberis, Boraginaceae, Bredia, Broussonetia, Caprifoliaceae, Celtis, Cornaceae, Cornus, } \\
\text { Corylopsis, Daphne, Desmodium, Euphorbiaceae, Euptelea, Fontanesia, Gleditsia, Guttiferae, Hamamelidaceae, } \\
\text { Hamamelis, Helwingia, Hydrangea, Hypericum, Koelreuteria, Lagerstroemia, Lauraceae, Leguminosae, } \\
\text { Lespedeza, Linaceae, Lonicera, Lythraceae, Malvaceae, Melia, Meliaceae, Meliosma, Mimosa, Mimosaceae, } \\
\text { Moraceae, Morus, Photinia, Phyllanthus, Pistacia, Prunus, Rhamnaceae, Rhamnus, Rhus, Ricinus, Rosaceae, } \\
\text { Rubus, Sambucus, Sapium, Schisandra, Schoepfia, Simaroubaceae, Sorbaria, Sorbus, Spiraea, Sterculiaceae, } \\
\text { Styracaceae, Styrax, Thymelaeaceae, Toxicodendron, Urticaceae, Verbenaceae, Violaceae, Vitex, Weigela, } \\
\text { Wikstroemia, Zanthoxylum, Zelkova, Ziziphus }\end{array}$ \\
\hline tr-m.dd.mb.lhs & $\begin{array}{l}\text { Tropical mesic drought-deciduous malacophyll } \\
\text { low and high shrub }\end{array}$ & $\begin{array}{l}\text { Acalypha, Acanthaceae, Albizia, Allophylus, Anacardiaceae, Artemisia, Asteroideae, Caesalpinia, } \\
\text { Capparidaceae, Caprifoliaceae, Compositae, Euphorbia, Euphorbiaceae, Flacourtiaceae, Helicteres, Labiatae, } \\
\text { Malvaceae, Microcos, Mussaenda, Rhamnaceae, Rhamnus, Rubiaceae, Rutaceae, Sapium, Simaroubaceae, } \\
\text { Styracaceae, Trema }\end{array}$ \\
\hline tr-x.dd.mb.lhs & $\begin{array}{l}\text { Tropical xeric drought-deciduous malacophyll } \\
\text { low and high shrub }\end{array}$ & $\begin{array}{l}\text { Acacia, Artemisia, Asteroideae, Calligonum, Capparidaceae, Capparis, Compositae, Cruciferae, Euphorbia, } \\
\text { Euphorbiaceae, Flacourtia, Flacourtiaceae, Leguminosae, Papilionaceae, Plumbaginaceae, Polygonaceae, } \\
\text { Rhamnaceae, Rubiaceae, Rutaceae, Simaroubaceae, Solanaceae, Solanum, Ziziphus }\end{array}$ \\
\hline ar.e.mb.eds & $\begin{array}{l}\text { Arctic evergreen malacophyll broad-leaved erect } \\
\text { dwarf shrub }\end{array}$ & Berberidaceae, Empetrum, Vaccinium, Vaccinium uliginosum \\
\hline
\end{tabular}


Table 1 (continued)

\begin{tabular}{|c|c|c|}
\hline PFT code & PFT name & Pollen taxa \\
\hline ar.cd.mb.eds & $\begin{array}{l}\text { Arctic cold-deciduous malacophyll broad-leaved } \\
\text { erect dwarf shrub }\end{array}$ & Betula, Betula middendorfii, Betulaceae, Caragana, Hippophae, Rhododendron, Rosa, Rosaceae \\
\hline ar.e.n.pds & Arctic evergreen need-leaved prostrate dwarf shrub & Juniperus, Sabina \\
\hline ar.e.mb.pds & $\begin{array}{l}\text { Arctic evergreen malacophyll broad-leaved } \\
\text { prostrate dwarf shrub }\end{array}$ & Oxycoccus, Rosaceae \\
\hline ar.cd.mb.pds & $\begin{array}{l}\text { Arctic cold-deciduous malacophyll broad-leaved } \\
\text { prostrate dwarf shrub }\end{array}$ & Betula, Betulaceae, Ceratoides, Rhododendron, Rosa, Rosaceae, Salicaceae, Salix \\
\hline cs & Cushion shrub & Ajania, Ceratoides, Nanophyton, Oxytropis, Oxytropis aciphylla, Plumbaginaceae, Salsola \\
\hline wt-dt.lv* & Warm-temperate drought-tolerant liana or vine & Dalbergia \\
\hline tr-dt.lv & Tropical drought-tolerant liana or vine & $\begin{array}{l}\text { Acacia, Albizia, Asclepiadaceae, Bowringia, Caesalpinia, Calamus, Capparidaceae, Capparis, Dalbergia, } \\
\text { Euphorbiaceae, Ficus, Piper, Piperaceae, Rubiaceae, Rutaceae, Vitaceae }\end{array}$ \\
\hline te-di.lv* & Temperate drought-intolerate liana or vine & $\begin{array}{l}\text { Actinidia, Asteroideae, Bignoniaceae, Capparidaceae, Celastraceae, Celastrus, Cocculus, Compositae, Hedera, } \\
\text { Lardizabalaceae, Leguminosae, Lonicera, Menispermaceae, Oleaceae, Papilionaceae, Ranunculaceae, } \\
\text { Rhamnaceae, Rosaceae, Rubus, Sabia, Sabiaceae, Schisandra, Vitaceae, Vitis, Zanthoxylum }\end{array}$ \\
\hline wt-di.lv* & Warm-temperate drought-intolerant liana or vine & $\begin{array}{l}\text { Actinidia, Aeschynanthus, Alyxia, Anodendron, Apocynaceae, Bignoniaceae, Broussonetia, Capparidaceae, } \\
\text { Celastraceae, Celastrus, Cocculus, Hedera, Lardizabalaceae, Leguminosae, Lonicera, Menispermaceae, } \\
\text { Myrsinaceae, Oleaceae, Papilionaceae, Porana, Ranunculaceae, Rubus, Sabia, Sabiaceae }\end{array}$ \\
\hline tr-di.lv* & Tropical drought-intolerant liana or vine & $\begin{array}{l}\text { Acanthaceae, Actinidia, Aeschynanthus, Aganosma, Alyxia, Annonaceae, Anodendron, Apocynaceae, } \\
\text { Asclepiadaceae, Bignoniaceae, Bridelia, Celastraceae, Celastrus, Cocculus, Combretaceae, Convolvulaceae, } \\
\text { Hedera, Hedyotis, Icacinaceae, Lardizabalaceae, Leguminosae, Mallotus, Mappianthus, Melastomataceae, } \\
\text { Menispermaceae, Merremia, Moraceae, Morinda, Mussaenda, Myrsinaceae, Nyctaginaceae, Olacaceae, } \\
\text { Oleaceae, Pachygone, Palmae, Pandanaceae, Papilionaceae, Porana, Pterolobium, Ranunculaceae, Rhynchodia, } \\
\text { Rubus, Sabia, Sabiaceae, Schefflera, Schisandra, Schizomussaenda, Simaroubaceae, Stephania, Sterculiaceae, } \\
\text { Tristellateia, Verbenaceae }\end{array}$ \\
\hline man* & Mangrove & $\begin{array}{l}\text { Acanthaceae, Acanthus ilicifolius, Aegiceras, Aegiceras corniculatum, Avicennia marina, Bignoniaceae, } \\
\text { Bruguiera, Bruguiera gymnorrhiza, Ceriops, Euphorbiaceae, Excoecaria, Excoecaria agallocha, Kandelia, } \\
\text { Kandelia candel, Lumnitzera racemosa, Myrsinaceae, Rhizophora, Rhizophora apiculata, Rhizophora stylosa, } \\
\text { Rhizophoraceae, Rubiaceae }\end{array}$ \\
\hline wpa* & Woody parasite & Arceuthobium, Elytranthe, Loranthaceae, Loranthus \\
\hline te-di.c* & Temperate drought-intolerant climber & $\begin{array}{l}\text { Amaranthaceae, Araliaceae, Campanulaceae, Cannabaceae, Clematis, Convolvulaceae, Convolvulus, } \\
\text { Cucurbitaceae, Cuscuta, Galium, Humulus, Leguminosae, Liliaceae, Mimosa, Mimosaceae, Papilionaceae, } \\
\text { Polygonaceae, Rubia, Rubiaceae, Solanaceae, Solanum, Trichosanthes, Valeriana, Valerianaceae, Vigna }\end{array}$ \\
\hline wt-di.c* & Warm-temperate drought-intolerant climber & $\begin{array}{l}\text { Acanthaceae, Amaranthaceae, Araliaceae, Campanulaceae, Convolvulaceae, Convolvulus, Cucurbitaceae, } \\
\text { Cuscuta, Galium, Leguminosae, Liliaceae, Mimosa, Mimosaceae, Papilionaceae, Rubia, Rubiaceae, Solanaceae, } \\
\text { Solanum, Trichosanthes, Vigna }\end{array}$ \\
\hline tr-di.c* & Tropical drought-intolerant climber & $\begin{array}{l}\text { Acanthaceae, Amaranthaceae, Araceae, Araliaceae, Asclepiadaceae, Campanulaceae, Convolvulaceae, } \\
\text { Convolvulus, Cucurbitaceae, Galium, Lathyrus, Lauraceae, Leguminosae, Liliaceae, Merremia, Mimosa, } \\
\text { Mimosaceae, Papilionaceae, Rubia, Rubiaceae, Sapindaceae, Solanaceae, Solanum, Trichosanthes, Vigna }\end{array}$ \\
\hline ar.fb & Arctic forb & $\begin{array}{l}\text { Androsace, Anemone, Arabis, Arenaria, Artemisia, Aster, Asteroideae, Astragalus, Bupleurum, Campanula, } \\
\text { Campanulaceae, Carduoideae, Caryophyllaceae, Centaurea, Cerastium, Compositae, Corydalis, Cruciferae, } \\
\text { Dianthus, Dipsacaceae, Dipsacus, Gentiana, Gentianaceae, Geraniaceae, Hypecoum, Impatiens, Incarvillea, } \\
\text { Iridaceae, Iris, Labiatae, Lamium, Leguminosae, Liguliflorae, Liliaceae, Liquiliflora, Lysimachia, Lythrum, } \\
\text { Mazus, Morina, Onobrychis, Orostachys, Oxytropis, Papaveraceae, Papilionaceae, Parnassia, Pedicularis, } \\
\text { Plumbaginaceae, Polemonium, Polygonaceae, Polygonum, Potentilla, Primula, Primulaceae, Pulsatilla, Pyrola, } \\
\text { Pyrolaceae, Ranunculaceae, Rhodiola, Saussurea, Saxifraga, Saxifragaceae, Scrophulariaceae, Sedum, } \\
\text { Serratula, Sibbaldia, Souliea, Thalictrum, Umbelliferae, Verbascum, Veronica }\end{array}$ \\
\hline bo-di.fb* & Boreal drought-intolerant forb & $\begin{array}{l}\text { Aconitum, Caryophyllaceae, Centaurea, Epilobium, Euphorbia, Euphorbiaceae, Filipendula, Hypecoum, Lathyrus, } \\
\text { Melilotus, Onobrychis, Pedicularis, Plantaginaceae, Plantago, Platycodon, Pyrola, Pyrolaceae, Scrophulariaceae }\end{array}$ \\
\hline te-di.fb* & Temperate drought-intolerant forb & $\begin{array}{l}\text { Acalypha, Aconitum, Amaranthaceae, Amaranthus, Ambrosia, Androsace, Anemone, Anthemis, Aquilegia, } \\
\text { Arabis, Araceae, Aralia, Araliaceae, Arctium, Arisaema, Artemisia, Asclepiadaceae, Asperula, Aster, Asteroideae, } \\
\text { Astragalus, Atractylodes, Berberidaceae, Boraginaceae, Bupleurum, Caltha, Campanula, Campanulaceae, } \\
\text { Cannabaceae, Caprifoliaceae, Carduoideae, Caryophyllaceae, Cassia, Centaurea, Cerastium, Chamaenerion, } \\
\text { Chamaenerion angustifolium, Chelidonium, Chenopodiaceae, Chenopodium, Chloranthus, Chrysanthemum, } \\
\text { Chrysosplenium, Cichorium, Circaea, Cirsium, Clematis, Commelinaceae, Compositae, Convolvulaceae, } \\
\text { Convolvulus, Cornaceae, Corydalis, Cruciferae, Dianthus, Dipsacaceae, Dipsacus, Droseraceae, Elsholtzia, } \\
\text { Epilobium, Euphorbia, Euphorbiaceae, Filipendula, Fragaria, Galium, Gentiana, Gentianaceae, Geraniaceae, } \\
\text { Geranium, Glycyrrhiza, Guttiferae, Hedysarum, Hemerocallis, Hypericum, Impatiens, Iridaceae, Iris, Ixeris, } \\
\text { Kochia, Labiatae, Lamium, Lathyrus, Leguminosae, Lespedeza, Ligularia, Liguliflorae, Liliaceae, Lilium, } \\
\text { Linaceae, Linum, Liquiliflora, Lysimachia, Lythraceae, Lythrum, Macleaya, Malvaceae, Mazus, Medicago, } \\
\text { Melilotus, Mimosa, Mimosaceae, Morina, Onagraceae, Onobrychis, Origanum, Oxalis, Oxytropis, Papaver, } \\
\text { Papaveraceae, Papilionaceae, Parnassia, Pedicularis, Phlomis, Plantaginaceae, Plantago, Platycodon, } \\
\text { Polemonium, Polygala, Polygonaceae, Polygonum, Polygonum bistorta, Polygonum viviparum, Potentilla, } \\
\text { Primula, Primulaceae, Pulsatilla, Pyrola, Pyrolaceae, Ranunculaceae, Ranunculus, Rheum, Ricinus, Rubia, } \\
\text { Rubiaceae, Rumex, Sanguisorba, Saururaceae, Saururus, Saussurea, Saxifraga, Saxifragaceae, Scabiosa, } \\
\text { Scrophulariaceae, Serratula, Solanaceae, Solanum, Stellera, Taraxacum, Thalictrum, Thymelaeaceae, Tribulus, } \\
\text { Trollius, Umbelliferae, Urtica, Urticaceae, Valeriana, Valerianaceae, Verbenaceae, Veronica, Viola, Violaceae, } \\
\text { Xanthium, Zygophyllaceae }\end{array}$ \\
\hline wt-di.fb* & Warm-temperate drought-intolerant forb & $\begin{array}{l}\text { Acalypha, Acanthaceae, Aconitum, Alocasia, Amaranthaceae, Amaranthus, Apocynaceae, Arabis, Araceae, } \\
\text { Araliaceae, Arisaema, Artemisia, Asclepiadaceae, Aster, Asteroideae, Astragalus, Atractylodes, Boraginaceae, } \\
\text { Bredia, Bupleurum, Campanula, Campanulaceae, Caprifoliaceae, Caryophyllaceae, Cassia, Chamaenerion, } \\
\text { Chelidonium, Chenopodiaceae, Chenopodium, Chloranthus, Chrysanthemum, Circaea, Cirsium, Clematis, } \\
\text { Clerodendrum, Commelinaceae, Compositae, Convolvulus, Corydalis, Cruciferae, Desmodium, Dianthus, } \\
\text { Dipsacaceae, Dipsacus, Droseraceae, Elsholtzia, Eomecon, Epilobium, Euphorbia, Euphorbiaceae, Fragaria, } \\
\text { Galium, Gentiana, Gentianaceae, Geraniaceae, Geranium, Gesneriaceae, Glycyrrhiza, Guttiferae, Hedyotis, } \\
\text { Hemerocallis, Hypericum, Impatiens, Iridaceae, Iris, Ixeris, Labiatae, Lamium, Lathyrus, Leguminosae, } \\
\text { Lespedeza, Ligularia, Liguliflorae, Liliaceae, Lilium, Linaceae, Lobelia, Lysimachia, Lythraceae, Lythrum, } \\
\text { Malvaceae, Mazus, Medicago, Melastomataceae, Melilotus, Mercurialis, Mimosa, Mimosaceae, Morina, }\end{array}$ \\
\hline
\end{tabular}


Table 1 (continued)

\begin{tabular}{|c|c|c|}
\hline PFT code & PFT name & Pollen taxa \\
\hline & & $\begin{array}{l}\text { Onagraceae, Ophiorrhiza, Oxalis, Papaveraceae, Papilionaceae, Parnassia, Pedicularis, Phlomis, } \\
\text { Plantaginaceae, Plantago, Platycodon, Polygala, Polygonaceae, Polygonum, Potentilla, Primula, Primulaceae, } \\
\text { Pyrola, Pyrolaceae, Ranunculaceae, Ranunculus, Rheum, Rostellularia, Rubia, Rubiaceae, Rumex, Sanguisorba, } \\
\text { Saururaceae, Saururus, Saussurea, Saxifraga, Saxifragaceae, Scrophulariaceae, Sesamum, Solanaceae, } \\
\text { Solanum, Stellera, Taraxacum, Thalictrum, Tropaeolaceae, Umbelliferae, Urtica, Urticaceae, Valeriana, } \\
\text { Valerianaceae, Verbenaceae, Vigna, Viola, Violaceae, Xanthium }\end{array}$ \\
\hline tr-di.fb* & Tropical drought-intolerant forb & $\begin{array}{l}\text { Acalypha, Acanthaceae, Aizoaceae, Alocasia, Amaranthaceae, Amaranthus, Anagallis, Apocynaceae, Araceae, } \\
\text { Araliaceae, Argemone, Argostemma, Arisaema, Artemisia, Asclepiadaceae, Aster, Asteroideae, Berberidaceae, } \\
\text { Boraginaceae, Campanulaceae, Capparidaceae, Caryophyllaceae, Cassia, Chenopodiaceae, Chenopodium, } \\
\text { Chingiacanthus, Chloranthus, Chrysanthemum, Cirsium, Clematis, Commelinaceae, Compositae, Convolvulus, } \\
\text { Corydalis, Cruciferae, Desmodium, Dianthus, Droseraceae, Eranthemum, Euphorbia, Euphorbiaceae, Galium, } \\
\text { Gentiana, Gentianaceae, Geraniaceae, Geranium, Gesneriaceae, Hedyotis, Hemiphragma, Impatiens, Iridaceae, } \\
\text { Iris, Ixeris, Justicia, Labiatae, Lathyrus, Leguminosae, Liguliflorae, Liliaceae, Lilium, Lobelia, Lythraceae, } \\
\text { Malvaceae, Melastomataceae, Melilotus, Mercurialis, Moraceae, Onagraceae, Ophiorrhiza, Oxalis, } \\
\text { Papilionaceae, Phlomis, Phyllanthus, Piper, Piperaceae, Plantaginaceae, Plantago, Platycodon, Polygala, } \\
\text { Polygonaceae, Polygonum, Potentilla, Primula, Primulaceae, Proteaceae, Ranunculaceae, Ranunculus, Reseda, } \\
\text { Rostellularia, Rubia, Rubiaceae, Rumex, Rungia, Saussurea, Saxifraga, Saxifragaceae, Scrophulariaceae, } \\
\text { Sesamum, Solanaceae, Solanum, Taraxacum, Thalictrum, Tiliaceae, Umbelliferae, Urticaceae, Verbenaceae, } \\
\text { Veronica, Vigna, Viola, Violaceae }\end{array}$ \\
\hline te-dt.fb & Temperate drought-tolerant forb & $\begin{array}{l}\text { Aconitum, Amaranthaceae, Ambrosia, Androsace, Anthemis, Arabis, Arenaria, Artemisia, Aster, Asteroideae, } \\
\text { Astragalus, Atractylodes, Atriplex, Bignoniaceae, Boraginaceae, Campanulaceae, Carduoideae, } \\
\text { Caryophyllaceae, Centaurea, Cerastium, Cichorium, Cirsium, Compositae, Convolvulaceae, Cruciferae, } \\
\text { Dianthus, Dipsacaceae, Echium, Filifolium, Filipendula, Gentiana, Gentianaceae, Geraniaceae, Hedysarum, } \\
\text { Hemerocallis, Hypecoum, Incarvillea, Iridaceae, Iris, Ixeris, Kochia, Labiatae, Leguminosae, Lespedeza, } \\
\text { Liguliflorae, Liliaceae, Lilium, Linaceae, Liquiliflora, Medicago, Onobrychis, Orobanche, Oxytropis, Papaver, } \\
\text { Papaveraceae, Papilionaceae, Peganum, Phlomis, Plumbaginaceae, Polemonium, Polygonaceae, Polygonum, } \\
\text { Potentilla, Primula, Primulaceae, Pulsatilla, Rosaceae, Rutaceae, Salsola, Saussurea, Saxifraga, Saxifragaceae, } \\
\text { Scrophulariaceae, Serratula, Sibbaldia, Solanaceae, Sophora alopecuroides, Stellera, Thalictrum, Thellungiella, } \\
\text { Thymus, Tribulus, Umbelliferae, Verbascum, Veronica, Viola, Violaceae, Xanthium, Zygophyllaceae, } \\
\text { Zygophyllum }\end{array}$ \\
\hline eu-dt.fb & Eurythermic drought-tolerant forb & Allium, Amaryllidaceae, Atriplex, Chenopodiaceae, Chenopodium, Limonium, Nyctaginaceae, Plumbaginaceae \\
\hline rc.fb & Rosette or cushion forb & $\begin{array}{l}\text { Ajania, Androsace, Arenaria, Asteroideae, Caryophyllaceae, Cerastium, Compositae, Dipsacaceae, Gentiana, } \\
\text { Gentianaceae, Geraniaceae, Leguminosae, Mazus, Oxytropis, Papaveraceae, Papilionaceae, Plumbaginaceae, } \\
\text { Rhodiola, Saussurea, Saxifraga, Saxifragaceae }\end{array}$ \\
\hline ha & Halophyte & Chenopodiaceae, Chenopodium, Salsola, Thellungiella \\
\hline hy* & Hydrophyte & $\begin{array}{l}\text { Acorus, Caltha, Haloragidaceae, Onagraceae, Phragmites, Ranunculaceae, Ranunculus, Saururaceae, Saururus, } \\
\text { Sparganiaceae, Sparganium, Thelypteridaceae, Thelypteris, Trollius }\end{array}$ \\
\hline $\mathrm{aq}^{*}$ & Aquatic & $\begin{array}{l}\text { Acorus, Alisma, Alismataceae, Araceae, Butomus, Ceratopteris, Droseraceae, Equisetum, Equisetum heleocharis, } \\
\text { Haloragidaceae, Hydrocharitaceae, Menyanthes, Myriophyllum, Nelumbo, Nuphar, Nymphaeaceae, } \\
\text { Nymphoides, Parkeriaceae, Phragmites, Polygonum, Potamogeton, Potamogetonaceae, Ranunculaceae, } \\
\text { Ranunculus, Salvinia, Sparganiaceae, Sparganium, Typha, Typhaceae }\end{array}$ \\
\hline g & Grass & Gramineae, Oryza sativa, Stipa \\
\hline s & Sedge & Carex, Cyperaceae, Cyperus, Scirpus \\
\hline$r^{*}$ & Rush & Juncaceae \\
\hline geo* & Geophyte & Allium, Amaryllidaceae, Araceae, Arisaema, Convolvulaceae, Cyperaceae, Iridaceae, Iris, Liliaceae, Lilium \\
\hline ssuc & Stem succulent & Cactaceae, Chrysosplenium, Euphorbia, Euphorbiaceae \\
\hline lsuc & Leaf succulent & Aizoaceae, Chenopodiaceae, Chenopodium, Crassulaceae, Orostachys, Rhodiola, Sedum \\
\hline ar.f* & Arctic fern or fern ally & Athyrium, Botrychium, Lunathyrium, Lycopodiaceae, Lycopodium \\
\hline eu.f* & Eurythermic fern or fern ally & $\begin{array}{l}\text { Adiantaceae, Adiantum, Athyriaceae, Athyrium, Botrychium, Coniogramme, Davallia, Davalliaceae, } \\
\text { Dennstaedtia, Dennstaedtiaceae, Dryopteridaceae, Dryopteris, Equisetum, Equisetum heleocharis, Filicales, } \\
\text { Gymnopteris, Hemionitidaceae, Hymenophyllaceae, Hymenophyllum, Lepisorus, Lycopodiaceae, Lycopodium, } \\
\text { Lycopodium serratum, Lygodiaceae, Lygodium, Onychium, Ophioglossum, Osmunda, Osmundaceae, } \\
\text { Polypodiaceae, Polypodium, Pteridaceae, Pteridium, Pteris, Pyrrosia, Salvinia, Selaginella, Selaginella sinensis, } \\
\text { Sinopteridaceae, Sinopteris, Thelypteridaceae, Thelypteris }\end{array}$ \\
\hline tr.f* & Tropical fern or fern ally & $\begin{array}{l}\text { Angiopteridaceae, Angiopteris, Antrophyum, Bauhinia, Botrychium, Ceratopteris, Cibotium, Cibotium barometz, } \\
\text { Dicranopteris, Gleichenia, Gleicheniaceae, Hemionitidaceae, Hicriopteris, Histiopteris, Lindsaea, } \\
\text { Lycopodiaceae, Lycopodium, Lygodiaceae, Lygodium, Microlepia, Osmundaceae, Parathelypteris, } \\
\text { Parkeriaceae, Pellaea, Phymatosorus, Plagiogyria, Plagiogyriaceae, Pteridaceae, Pteris, Schizaea, Vittaria }\end{array}$ \\
\hline $\mathrm{tf}$ & Tree fern & Alsophila, Cyatheaceae \\
\hline epi* & Epiphyte & No taxa \\
\hline rps* & Root parasite & Cuscuta, Lauraceae, Loranthaceae, Monotropa, Orobanche \\
\hline $\mathrm{m}^{*}$ & Moss & Sphagnum \\
\hline
\end{tabular}

while collecting pollen samples. In sites where modern vegetation information was not available, it was simply recorded (e.g. only forest, grassland, or shrubland, without any species composition), or where vegetation data were ambiguous, information derived from the digitized vegetation map of China at 1:1 million scale (EBVAC, 2001) was used to define the biomes in conjunction with observed vegetation records from surrounding sites. Wherever agricultural vegetation has been mapped onto a site or the mapped vegetation is considered to be incorrect, we have usually used the potential natural vegetation based on bioclimatic information and the observed vegetation from surrounding sites. The observed "real" modern biomes were then assigned (Fig. 2a; Appendix A) according to the observed and mapped vegetation types as well as the principle vegetation of China (ECVC, 1980; EBVAC, 2001) and biome characteristics (Table 2).

The surface pollen assemblages not only reflect the natural vegetation but also vegetation affected by human activity and land use. Therefore the pollen-based reconstruction of modern biomes incorporates information on anthropogenic biomes. The observed biomes also reflect information from natural and disturbed vegetation. Comparison between the observed and reconstructed biomes therefore implies a level of disturbance by human activity. However 
Table 2

Characteristics of Chinese biomes.

\begin{tabular}{|c|c|c|c|c|c|}
\hline Biome & Structure & Dominant PFT(s) & Characteristic taxa & $\begin{array}{l}\text { Terminology used in } \\
\text { the Chinese literature } \\
\text { for equivalents }\end{array}$ & Distribution in China \\
\hline Cold deciduous forest & $\begin{array}{l}\text { More or less open canopy } \\
\text { forest, medium to tall cold- } \\
\text { deciduous malacophyll } \\
\text { broad-leaved trees, with a } \\
\text { rich understorey of shrubs, } \\
\text { forbs and mosses in moist } \\
\text { habitats or a depauperate } \\
\text { understorey of shrubs and } \\
\text { lichens in dry habitats }\end{array}$ & $\begin{array}{l}\text { Boreal deciduous needle- } \\
\text { leaved trees, boreal cold- } \\
\text { deciduous malacophyll } \\
\text { broad-leaved trees }\end{array}$ & Larix, Betula & $\begin{array}{l}\text { Cold-temperate } \\
\text { deciduous needle- } \\
\text { leaved forest }\end{array}$ & $\begin{array}{l}\text { northern Daxingan } \\
\text { Mountains, Tianshan and } \\
\text { Altai Mountains and } \\
\text { subalpine regions around } \\
\text { the southeastern Tibetan } \\
\text { Plateau }\end{array}$ \\
\hline $\begin{array}{l}\text { Cold evergreen } \\
\text { needle-leaved forest }\end{array}$ & $\begin{array}{l}\text { Closed canopy forest of } \\
\text { evergreen needle-leaved } \\
\text { trees with a rich } \\
\text { understorey of shrubs, forbs } \\
\text { and mosses }\end{array}$ & $\begin{array}{l}\text { Boreal evergreen needle- } \\
\text { leaved trees }\end{array}$ & $\begin{array}{l}\text { Abies sibirica, A. fabri, Picea } \\
\text { obovata, P. purpurea }\end{array}$ & $\begin{array}{l}\text { Cold-temperate } \\
\text { evergreen needle- } \\
\text { leaved forest; taiga }\end{array}$ & $\begin{array}{l}\text { Daxingan and Changbai } \\
\text { Mountains; Wutai, Yan, } \\
\text { Luliang and Taihang } \\
\text { Mountains; Qinling and } \\
\text { Daba Mountains; Altai, } \\
\text { Tianshan, Qilian, Helan and } \\
\text { Yin Mountains; and eastern } \\
\text { and southern slopes of the } \\
\text { Tibetan Plateau, 1100- } \\
4300 \mathrm{~m}\end{array}$ \\
\hline $\begin{array}{l}\text { Cool-temperate evergreen } \\
\text { needle-leaved and } \\
\text { mixed forest }\end{array}$ & $\begin{array}{l}\text { Closed to open canopy forest } \\
\text { of evergreen needle-leaved } \\
\text { trees and deciduous broad- } \\
\text { leaved trees }\end{array}$ & $\begin{array}{l}\text { Cool-temperate evergreen } \\
\text { needle-leaved trees, boreal } \\
\text { cold-deciduous malacophyll } \\
\text { broad-leaved trees }\end{array}$ & $\begin{array}{l}\text { Pinus sylvestris var. } \\
\text { mongolica, Betula, Populus }\end{array}$ & Cold mixed forest & $\begin{array}{l}\text { High mountains in } \\
\text { northeastern, western and } \\
\text { southwestern China }\end{array}$ \\
\hline $\begin{array}{l}\text { Cool evergreen needle- } \\
\text { leaved forest }\end{array}$ & $\begin{array}{l}\text { Closed to open canopy forest } \\
\text { of evergreen needle-leaved } \\
\text { trees }\end{array}$ & $\begin{array}{l}\text { Boreal and cold-temperate } \\
\text { evergreen needle-leaved } \\
\text { trees }\end{array}$ & $\begin{array}{l}\text { Abies nephrolepis, Picea } \\
\text { jezoensis, } \text { P. schrenkiana, } \\
\text { Pinus, Sabina }\end{array}$ & Cool conifer forest & $\begin{array}{l}\text { Daxingan Mountains, Altai } \\
\text { Mountains, eastern high } \\
\text { slope of the Tibetan Plateau }\end{array}$ \\
\hline Cool mixed forest & $\begin{array}{l}\text { Closed to open canopy forest } \\
\text { with evergreen needle- } \\
\text { leaved trees and deciduous } \\
\text { broad-leaved trees }\end{array}$ & $\begin{array}{l}\text { Boreal and cold-temperate } \\
\text { evergreen needle-leaved } \\
\text { trees, temperate spring-frost } \\
\text { tolerant cold-deciduous } \\
\text { malacophyll broad-leaved } \\
\text { trees }\end{array}$ & $\begin{array}{l}\text { Pinus koraiensis, Larix, Abies, } \\
\text { Picea, Betula, Tilia, Fraxinus, } \\
\text { Acer (northeast China); } \\
\text { Tsuga, Abies, Picea, Acer, } \\
\text { Betula, Pinus (high } \\
\text { mountains in southwest } \\
\text { China) }\end{array}$ & $\begin{array}{l}\text { Cool-temperate } \\
\text { mixed conifer broad- } \\
\text { leaved forest }\end{array}$ & $\begin{array}{l}\text { Changbai, Xiaoxingan and } \\
\text { Zhangguangcai Mountains; } \\
\text { high mountains in } \\
\text { southwestern China and } \\
\text { southern Tibetan Plateau }\end{array}$ \\
\hline $\begin{array}{l}\text { Temperate deciduous } \\
\text { broad-leaved forest }\end{array}$ & $\begin{array}{l}\text { More or less open canopy } \\
\text { forest (ground cover } \\
\text { between } 40 \text { and } 70 \% \text { ) of } \\
\text { deciduous broad-leaved } \\
\text { trees }\end{array}$ & $\begin{array}{l}\text { Temperate spring-frost } \\
\text { avoiding and intolerant } \\
\text { cold-deciduous malacophyll } \\
\text { broad-leaved trees, } \\
\text { sometimes with temperate } \\
\text { evergreen needle-leaved } \\
\text { trees and eurythermic } \\
\text { evergreen needle-leaved } \\
\text { trees }\end{array}$ & $\begin{array}{l}\text { Quercus, Castanea, Acer, } \\
\text { Alnus, Ulmus, Populus, Pinus } \\
\text { tabbulaeformis, Pinus } \\
\text { densiflora, Platycladus }\end{array}$ & & $\begin{array}{l}\text { northern, central, } \\
\text { northwestern and } \\
\text { southwestern China }\end{array}$ \\
\hline $\begin{array}{l}\text { Warm-temperate evergreen } \\
\text { broad-leaved and mixed } \\
\text { forest }\end{array}$ & $\begin{array}{l}\text { Closed canopy forest of tall } \\
\text { malacophyll and sclerophyll } \\
\text { broad-leaved trees, with } \\
\text { deciduous broad-leaved } \\
\text { trees, and deciduous and } \\
\text { evergreen needle-leaved } \\
\text { trees }\end{array}$ & $\begin{array}{l}\text { Warm-temperate evergreen } \\
\text { malacophyll and sclerophyll } \\
\text { broad-leaved trees, } \\
\text { temperate spring-frost } \\
\text { intolerant cold-deciduous } \\
\text { malacophyll broad-leaved } \\
\text { trees, warm-temperate } \\
\text { evergreen and deciduous } \\
\text { needle-leaved trees }\end{array}$ & $\begin{array}{l}\text { Castanopsis, Lithocarpus, } \\
\text { Cyclobalanopsis, Schima, } \\
\text { Machilus, Cinnamomum, } \\
\text { Phoebe, Quercus, } \\
\text { Liquidamber, Platycarya, } \\
\text { Fagus, Metasequoia, } \\
\text { Glyptostrobus, Pseudolarix, } \\
\text { Taxodium, Pinus } \\
\text { (massoniana, yunnanensis, } \\
\text { khaya, griffithii), Keteleeria, } \\
\text { Cupressus, Cunninghamia }\end{array}$ & $\begin{array}{l}\text { Northern and central } \\
\text { subtropical evergreen } \\
\text { broad-leaved forest, } \\
\text { typical evergreen } \\
\text { broad-leaved forest }\end{array}$ & $\begin{array}{l}\text { southern China (northern to } \\
\text { central subtropical region) }\end{array}$ \\
\hline $\begin{array}{l}\text { Warm-temperate evergreen } \\
\text { broad-leaved forest }\end{array}$ & $\begin{array}{l}\text { Closed canopy forest of tall } \\
\text { warm-temperate evergreen } \\
\text { malacophyll and sclerophyll } \\
\text { broad-leaved trees in the } \\
\text { first layer, with sparse } \\
\text { warm-temperate deciduous } \\
\text { broad-leaved trees in the } \\
\text { second and third layers }\end{array}$ & $\begin{array}{l}\text { Warm-temperate evergreen } \\
\text { malacophyll and sclerophyll } \\
\text { broad-leaved trees }\end{array}$ & $\begin{array}{l}\text { Castanopsis, Cryptocarya, } \\
\text { Elaeocarpus, Engelhardtia, } \\
\text { Schima, Syzygium, } \\
\text { Lauraceae, Fagaceae, } \\
\text { evergreen Quercus } \\
\text { (aquifolioides, pannosa, } \\
\text { gilliana, spinosa, senescens, } \\
\text { spathulata) }\end{array}$ & $\begin{array}{l}\text { Monsoon evergreen } \\
\text { broad-leaved forest, } \\
\text { montane and river } \\
\text { valley sclerophyll } \\
\text { evergreen broad- } \\
\text { leaved forest }\end{array}$ & $\begin{array}{l}\text { southern China (southern } \\
\text { subtropical region); high } \\
\text { mountains and dry river } \\
\text { valley of southwestern } \\
\text { China and southeastern } \\
\text { Tibetan Plateau }\end{array}$ \\
\hline $\begin{array}{l}\text { Tropical semi-evergreen } \\
\text { broad-leaved forest }\end{array}$ & $\begin{array}{l}\text { Closed canopy forest } \\
\text { dominated by tropical mesic } \\
\text { drought-deciduous broad- } \\
\text { leaved trees with more or } \\
\text { less tropical evergreen } \\
\text { malacophyll broad-leaved } \\
\text { trees; some proportion of } \\
\text { the canopy leaves fall in the } \\
\text { dry season }\end{array}$ & $\begin{array}{l}\text { Tropical mesic drought- } \\
\text { deciduous malacophyll } \\
\text { broad-leaved trees, tropical } \\
\text { evergreen malacophyll } \\
\text { broad-leaved trees, tropical } \\
\text { evergreen malacophyll low } \\
\text { to high shrubs, drought- } \\
\text { tolerant lianas and vines }\end{array}$ & $\begin{array}{l}\text { Ficus, Gironniera, Syzygium, } \\
\text { Steculia, Vatica, Parashorea, } \\
\text { Burretiodendron }\end{array}$ & $\begin{array}{l}\text { Semi-evergreen } \\
\text { seasonal forest; } \\
\text { tropical seasonal } \\
\text { forest }\end{array}$ & $\begin{array}{l}\text { southern China (south of the } \\
\text { Tropic of Cancer) and } \\
\text { southern slope of Himalayan } \\
\text { Ranges }\end{array}$ \\
\hline $\begin{array}{l}\text { Tropical evergreen broad- } \\
\text { leaved forest }\end{array}$ & $\begin{array}{l}\text { Closed canopy forest with } \\
\text { tall tropical evergreen } \\
\text { broad-leaved malacophyll }\end{array}$ & $\begin{array}{l}\text { Tropical evergreen } \\
\text { sclerophyll and malacophyll } \\
\text { broad-leaved trees, tropical }\end{array}$ & $\begin{array}{l}\text { Myristica, Vatica, Hopea, } \\
\text { Dipterocarpus, Terminalia, } \\
\text { Pometia }\end{array}$ & Tropical rainforest & $\begin{array}{l}\text { southernmost mainland of } \\
\text { China, southern Taiwan } \\
\text { Island and Hainan Island }\end{array}$ \\
\hline
\end{tabular}


Table 2 (continued)

\begin{tabular}{|c|c|c|c|c|c|}
\hline Biome & Structure & Dominant PFT(s) & Characteristic taxa & $\begin{array}{l}\text { Terminology used in } \\
\text { the Chinese literature } \\
\text { for equivalents }\end{array}$ & Distribution in China \\
\hline & $\begin{array}{l}\text { and sclerophyll trees, } \\
\text { shrubs, lianas and vines }\end{array}$ & $\begin{array}{l}\text { evergreen malacophyll low } \\
\text { to high shrubs, drought } \\
\text { intolerant lianas and vines }\end{array}$ & & & \\
\hline $\begin{array}{l}\text { Tropical deciduous broad- } \\
\text { leaved forest and } \\
\text { woodland }\end{array}$ & $\begin{array}{l}\text { Open canopy forest } \\
\text { dominated by medium } \\
\text { tropical xeric drought- } \\
\text { deciduous trees with sparse } \\
\text { shrubs and herbs; most or } \\
\text { all the canopy leaves fall in } \\
\text { the dry season (5- } \\
7 \text { months) }\end{array}$ & $\begin{array}{l}\text { Tropical xeric drought- } \\
\text { deciduous malacophyll } \\
\text { broad-leaved trees, } \\
\text { eurythemic mesic drought- } \\
\text { deciduous malacophyll low } \\
\text { or high shrubs }\end{array}$ & $\begin{array}{l}\text { Bombax, Albizia, Terminalia, } \\
\text { Lannea }\end{array}$ & $\begin{array}{l}\text { Seasonal deciduous } \\
\text { forest }\end{array}$ & $\begin{array}{l}\text { hot and dry river vallies in } \\
\text { southern Yunnan Province, } \\
\text { and western Hainan Island }\end{array}$ \\
\hline $\begin{array}{l}\text { Temperate xerophytic } \\
\text { shrubland }\end{array}$ & $\begin{array}{l}\text { Sparse to open, clumped or } \\
\text { un-clumped vegetation } \\
\text { dominated by small-leaved } \\
\text { and micro-leaved small } \\
\text { trees and shrubs }\end{array}$ & $\begin{array}{l}\text { Small-leaved and micro- } \\
\text { leaved trees and low/high } \\
\text { shrubs, temperate drought- } \\
\text { tolerant forbs }\end{array}$ & $\begin{array}{l}\text { Artemisia, Ephedra, } \\
\text { Chenopodiaceae, Caragana }\end{array}$ & Forested dry steppe & northern to western China \\
\hline Temperate grassland & $\begin{array}{l}\text { Dense to sparse treeless } \\
\text { vegetation dominated by } \\
\text { graminoids and forbs with } \\
\text { few shrubs }\end{array}$ & $\begin{array}{l}\text { Graminoids, temperate forbs } \\
\text { and shrubs }\end{array}$ & $\begin{array}{l}\text { Stipa, Leymus, Festuca, } \\
\text { Cleistogens, Agropyron, } \\
\text { Artemisia }\end{array}$ & $\begin{array}{l}\text { Typical (dry) steppe, } \\
\text { meadow steppe, } \\
\text { desert steppe }\end{array}$ & $\begin{array}{l}\text { northern and western China, } \\
\text { including the Tibetan } \\
\text { Plateau }\end{array}$ \\
\hline Desert & $\begin{array}{l}\text { Bare ground with sparse } \\
\text { grasses and shrubs }\end{array}$ & $\begin{array}{l}\text { Graminoids, temperate } \\
\text { drought-tolerant shrubs }\end{array}$ & $\begin{array}{l}\text { Haloxylon, Ephedra, } \\
\text { Zygophyllum, Nitraria, } \\
\text { Caragana, Calligonum, } \\
\text { Reaumuria, Salsola, } \\
\text { Sympegma, Artemisia, Ajania }\end{array}$ & $\begin{array}{l}\text { Little tree desert, } \\
\text { shrub desert, semi- } \\
\text { shrub desert }\end{array}$ & $\begin{array}{l}\text { western China, including the } \\
\text { Tibetan Plateau }\end{array}$ \\
\hline Cushion-forb tundra & $\begin{array}{l}\text { Discontinuous treeless } \\
\text { vegetation characterised by } \\
\text { rosette or cushion forbs, } \\
\text { grasses, mosses and lichens }\end{array}$ & $\begin{array}{l}\text { Rosette or cushion forbs, } \\
\text { graminoids }\end{array}$ & $\begin{array}{l}\text { Papaver, Oxytropis, Draba, } \\
\text { Saussurea, Caryophyllaceae, } \\
\text { Saxifragaceae, Rhodiola, } \\
\text { Carex }\end{array}$ & $\begin{array}{l}\text { High mountain } \\
\text { tundra }\end{array}$ & $\begin{array}{l}\text { Changbai Mountains, } \\
\text { Tianshan and Qilian } \\
\text { Mountains, eastern Tibetan } \\
\text { Plateau, Yulong Mountains }\end{array}$ \\
\hline Graminoid and forb tundra & $\begin{array}{l}\text { Treeless and predominantly } \\
\text { herbaceous vegetation } \\
\text { dominated by alpine forbs, } \\
\text { graminoids, and lichens }\end{array}$ & Alpine forbs, graminoids & $\begin{array}{l}\text { Kobresia, Stipa, Carex, } \\
\text { Polygonum, Gentiana, } \\
\text { Festuca, Artemisia }\end{array}$ & $\begin{array}{l}\text { High-cold meadow, } \\
\text { high-cold steppe }\end{array}$ & $\begin{array}{l}\text { Tibetan Plateau and its } \\
\text { periphery, Qilian, Tianshan, } \\
\text { Parmir, Taibai and } \\
\text { Xiaowutai Mountains }\end{array}$ \\
\hline $\begin{array}{l}\text { Prostrate dwarf-shrub } \\
\text { tundra }\end{array}$ & $\begin{array}{l}\text { Treeless vegetation } \\
\text { dominated by prostrate } \\
\text { dwarf shrubs, graminoids, } \\
\text { arctic forbs, true mosses and } \\
\text { lichens }\end{array}$ & $\begin{array}{l}\text { Alpine prostrate dwarf } \\
\text { shrubs, graminoids, arctic } \\
\text { forbs }\end{array}$ & $\begin{array}{l}\text { Dryas, Vaccinium, } \\
\text { Rhododendron } \\
\text { xanthastephonum, Salix } \\
\text { rotundifolia, Pedicularis, } \\
\text { Arenaria, Thylacospermum, } \\
\text { Androsace, Potentilla, } \\
\text { Sibbaldianthe, Acantholimon, } \\
\text { Oxytropis, Astrugalus, } \\
\text { Ceratoides }\end{array}$ & $\begin{array}{l}\text { High mountain dwarf } \\
\text { vegetation, high-cold } \\
\text { desert }\end{array}$ & $\begin{array}{l}\text { Changbai Mountains, Qilian } \\
\text { Mountain, Tibetan Plateau, } \\
\text { Tianshan Mountains }\end{array}$ \\
\hline Erect dwarf-shrub tundra & $\begin{array}{l}\text { Treeless vegetation } \\
\text { dominated by erect dwarf } \\
\text { shrubs, with grasses, true } \\
\text { mosses and lichens }\end{array}$ & $\begin{array}{l}\text { Erect dwarf shrubs, } \\
\text { graminoids }\end{array}$ & $\begin{array}{l}\text { Sabina, Juniperus, Dasiphora, } \\
\text { Empetrum, Vaccinium }\end{array}$ & $\begin{array}{l}\text { High-cold shrubland } \\
\text { (evergreen conifer } \\
\text { shrub) }\end{array}$ & $\begin{array}{l}\text { Altai and Tianshan } \\
\text { Mountains, Changbai } \\
\text { Mountains, Tibetan Plateau } \\
\text { and high mountains around } \\
\text { its periphery }\end{array}$ \\
\hline Low and high shrub tundra & $\begin{array}{l}\text { Treeless vegetation } \\
\text { dominated by deciduous or } \\
\text { evergreen low or high } \\
\text { shrubs, sometimes with } \\
\text { tussock-forming graminoids } \\
\text { and true mosses, bog } \\
\text { mosses, and lichens }\end{array}$ & $\begin{array}{l}\text { Deciduous or evergreen low } \\
\text { or high shrubs, graminoids }\end{array}$ & $\begin{array}{l}\text { Rhododendron capitatum, } \\
\text { Betula rotundifolia, Salix } \\
\text { oritrepha, S. cupularis, } \\
\text { Hippophae, Spiraea }\end{array}$ & $\begin{array}{l}\text { High-cold shrubland, } \\
\text { evergreen sclerophyll } \\
\text { shrubland, deciduous } \\
\text { broad-leaved } \\
\text { shrubland }\end{array}$ & $\begin{array}{l}\text { Tianshan, Altai and Qilian } \\
\text { Mountains, Changbai } \\
\text { Mountains, eastern Tibetan } \\
\text { Plateau, high mountains in } \\
\text { southwestern China, Taibai } \\
\text { Mountains, Yushan } \\
\text { Mountains }\end{array}$ \\
\hline
\end{tabular}

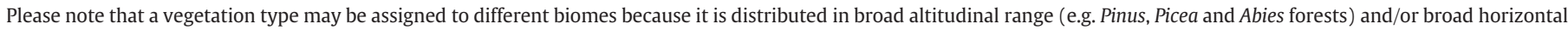
belt (e.g. many kinds of steppes, deserts and shrublands), but here it is only assigned to one biome due to insufficient information in the digitized vegetation map.

past vegetation reconstructed from pollen is usually expressed as potential natural biomes. Human disturbed biomes are concealed from the reconstruction. Therefore more information about the modern potential natural biomes (and also the anthropogenic biomes) is needed in order to better reconstruct biomes from pollen records.

For this purpose we used the potential natural biomes predicted by the BIOME4 global vegetation model with coupled biogeographical and biogeochemical processes (Kaplan, 2001; Kaplan et al., 2003). The model is driven by gridded monthly mean temperature, precipitation, cloudiness and minimum temperature, soil properties and a fixed $\mathrm{CO}_{2}$ concentration of $360 \mathrm{ppm}$. The original climate data was derived from averaged long-term observations at 1814 weather stations between 1971 and 2000 (China Meteorological Administration, unpublished). The data was interpolated into $10 \mathrm{~km}$ grid cells by the thin plate smoothing spline surface fitting technique (Hutchinson, 2006) on the basis of the STRM digital elevation model (Farr et al., 2007). The soil water holding capacity and percolation rate compiled by Kaplan (2001) were derived from a global digital soil map of the Food and Agriculture Organization (FAO) of the United Nations.

The model originally predicted 26 biomes in China, of which 19 appeared in surface pollen sampling sites (modeling grids), but such 19 simulated biomes do not match exactly with the 19 biomes used for biomization (Tables 2 and 3). The biomes used for biomization are derived to a great extent from BIOME4 model (Ni et al., 2010), so that we combined or separated some biomes from BIOME4 simulation to match the 19 biomes used for biomization based on their consistent definitions. On the other hand, some simulated biomes are distributed in fewer sites, for example the temperate conifer forest, evergreen and 
Table 3

Defining biomes of China using key plant functional types (PFTs).

\begin{tabular}{|c|c|c|}
\hline Biome code & Biome name & PFTs \\
\hline CLDE & Cold deciduous forest & bo.cd.mb.lhs, bo.cd.mb.t, bo.d.n.t, bo.e.mb.lhs, eu.e.n.t \\
\hline CLEG & Cold evergreen needle-leaved forest & bo.cd.mb.lhs, bo.cd.mb.t, bo.d.n.t, bo.e.mb.lhs, bo.e.n.t, eu.e.n.t \\
\hline CLMX & $\begin{array}{l}\text { Cold-temperate evergreen needle-leaved and mixed } \\
\text { forest }\end{array}$ & bo.cd.mb.lhs, bo.cd.mb.t, bo.d.n.t, bo.e.mb.lhs, c-te.e.n.t, eu.e.n.t \\
\hline COEG & Cool evergreen needle-leaved forest & bo.cd.mb.lhs, bo.cd.mb.t, bo.d.n.t, bo.e.mb.lhs, c-te.e.n.t, eu.e.n.t, te-ft.cd.mb.t \\
\hline COMX & Cool mixed forest & bo.d.n.t, bo.e.mb.lhs, c-te.e.n.t, eu.e.n.t, te.cd.mb.lhs, te-fa.cd.mb.t, te-ft.cd.mb.t \\
\hline TEDE & Temperate deciduous broad-leaved forest & bo.cd.mb.t, eu.e.n.t, te.cd.mb.lhs, te.e.n.t, te-fa.cd.mb.t, te-fi.cd.mb.t \\
\hline WTEM & $\begin{array}{l}\text { Warm-temperate evergreen broad-leaved and mixed } \\
\text { forest }\end{array}$ & $\begin{array}{l}\text { eu.e.n.t, te-fi.cd.mb.t, wt.cd.mb.lhs, wt.cd.mb.t, wt.d.n.t, wt.e.mb.lhs, wt.e.mb.t, wt.e.n.t, wt.e.sb.lhs, wt } \\
\text { e.sb.t }\end{array}$ \\
\hline WTEG & Warm-temperate evergreen broad-leaved forest & eu.e.n.t, tf, wt.e.mb.lhs, wt.e.mb.t, wt.e.n.t, wt.e.sb.lhs, wt.e.sb.t \\
\hline TRSE & Tropical semi-evergreen broad-leaved forest & tf, tr.e.mb.lhs, tr.e.mb.t, tr-dt.lv, tr-m.dd.mb.t, wt.d.n.t, wt.e.mb.t, wt.e.n.t, wt.e.sb.t \\
\hline TREG & Tropical evergreen broad-leaved forest & tf, tr.e.mb.lhs, tr.e.mb.t, tr.e.sb.t, tr-di.lv, tu.t, wt.d.n.t, wt.e.mb.t, wt.e.n.t, wt.e.sb.t \\
\hline TRDE & Tropical deciduous broad-leaved forest and woodland & g, tr-m.dd.mb.lhs, tr-m.dd.mb.t, tr-x.dd.mb.lhs, tr-x.dd.mb.t \\
\hline TEXE & Temperate xerophytic shrubland & dt.sl.lhs, g, ml.t, sl.t, te-dt.fb \\
\hline TEGR & Temperate grassland & di.sl.lhs, eu-dt.fb, g, s, te-dt.fb \\
\hline DESE & Desert & cs, dt.sl.lhs, ft.ml.lhs, g, ha, lsuc, ssuc \\
\hline CUSH & Cushion-forb tundra & ar.fb, g, rc.fb \\
\hline DRYT & Graminoid and forb tundra & ar.fb, $\mathrm{g}, \mathrm{s}$ \\
\hline PROS & Prostrate dwarf-shrub tundra & ar.cd.mb.pds, ar.e.mb.pds, ar.fb, g \\
\hline DWAR & Erect dwarf-shrub tundra & ar.cd.mb.eds, ar.cd.mb.pds, ar.e.mb.eds, ar.e.mb.pds, g, s \\
\hline SHRU & Low and high shrub tundra & ar.cd.mb.eds, ar.cd.mb.lhs, ar.cd.mb.pds, ar.e.mb.eds, ar.e.mb.lhs, ar.e.mb.pds, ar.e.n.lhs, ar.e.n.pds, g, s \\
\hline
\end{tabular}

deciduous taiga, and tropical savanna which can be assigned to close biomes with larger distributions. The simulated temperate deciduous forest and temperate conifer forest were combined to form a biome of temperate deciduous broad-leaved forest that in definition match the same biome represented in the pollen samples. The simulated warmtemperate mixed forest was separated by its latitudinal range into warm-temperate evergreen broad-leaved and mixed forest $\left(>24^{\circ} \mathrm{N}\right)$, and warm-temperate evergreen broad-leaved forest $\left(<24^{\circ} \mathrm{N}\right)$. Evergreen taiga/montane forest was combined with cold evergreen needle-leaved forest, deciduous taiga/montane forest with colddeciduous forest, and tropical savanna with tropical deciduous broad-leaved forest. Other simulated biomes are the same as for the biomization used (Tables 2 and 3 ).

\section{Results and discussion}

\subsection{Zonal biome reconstruction}

Pollen-based reconstruction shows a relatively clear spatial distribution of modern biomes throughout China (Fig. 2b). Site by site comparison with the vegetation observations (Fig. 2a) indicated a $68.8 \%$ agreement (Table 4), implying that the application of the biomization method to Chinese surface pollen records was successful.

\subsubsection{Biome distribution and comparison}

Cold-deciduous forest (CLDE) was only correctly reconstructed in 15 samples (26.8\%; Table 4) in mountainous areas of northeastern and northwestern China and in subalpine regions of southeastern Tibet (Fig. 2b). Other sites were wrongly assigned to cold evergreen needleleaved forest (CLEG), cool evergreen needle-leaved forest (COEG), and cool mixed forest (COMX) within similar areas as CLDE, and also to prostrate dwarf-shrub tundra (PROS) in northeastern China (Fig. 2b). Six COMX sites were incorrectly assigned to CLDE. The reconstructed CLDE also occurred in subtropical areas as a result of seven samples from warm-temperate evergreen broad-leaved and mixed forest (WTEM) in mountains being wrongly identified as CLDE (Fig. 2b and Table 4).

Samples correctly reconstructed as CLEG (57.4\%) mainly originate from the same mountainous areas as CLDE (Fig. 2b), which are geographically coincident with the actual vegetation (Fig. 2a). CLEG was also wrongly assigned to COMX and temperate xerophytic shrubland (TEXE), whilst COEG, COMX and temperate grassland (TEGR) were incorrectly identified as CLEG (Table 4).
There are only three samples of cold-temperate evergreen needleleaved and mixed forest (CLMX) which often coexists with CLDE and CLEG and occupies very small areas in northeastern China (Table 2). The biome was not correctly reconstructed (Table 4).

Modern biomes of COEG and COMX occur together in both northeastern China, on the eastern and northern Tibetan Plateau and in the high mountains in central and eastern China (Table 2). They were largely correctly reconstructed (64.4\% and 65\%, respectively; Table 4). COEG and COMX were incorrectly assigned in a few sites to CLEG, COMX, TEXE and TEGR, while some of CLDE, COMX and TEGR were wrongly identified as COEG and COMX (Table 4). Furthermore COMX was wrongly assigned to PROS in 17 sites and as temperate deciduous broad-leaved forest (TEDE) in seven sites (Fig. 2b and Table 4).

The incorrect assignment of cold- and cool-temperate forests results mainly from the fact that these biomes contain the same widespread needle-leaved pollen taxa (Abies, Picea, Pinus and Tsuga) and therefore the same key PFTs. Incorrect identification of forest biomes and some treeless biomes of grassland, shrubland and tundra are the likely result of two factors: the local existence of conifer trees in small patches and/or the long-distance transportation of conifer tree pollen from forested areas, and the share in forest and non-forest biomes of the same pollen taxa but assigned to different shrub PFTs used to differentiate different biomes. For example, Betula and Salix were assigned to boreal cold-deciduous malacophyll broad-leaved low and high shrub, and Rhododendra and Ledum to boreal evergreen malacophyll broad-leaved low and high shrub (Table 1), which were used to define several cold-temperate forests (Table 3). However Rhododendra and Ledum were also assigned to arctic evergreen malacophyll broad-leaved low and high shrub, and Betula and Rhododendra to arctic cold-deciduous malacophyll broad-leaved erect and prostrate dwarf shrubs (Table 1), which were used to define tundra biomes (Table 3 ). These led likely to wrong assignment between cold-temperate forests and tundra biomes.

$64.2 \%$ of samples of TEDE, which is widely distributed in northern, central, northwestern and southwestern China (Table 2), were correctly identified (Table 4 and Fig. 2b). Eight samples of TEDE were incorrectly assigned as cold-temperate forests, and seven COMX sites were wrongly identified as TEDE, in the Changbai Mountains where deciduous broad-leaved forest grows in mosaic patches with evergreen conifer and mixed forests (Table 2). 22 sites of TEDE were incorrectly classified as non-forest biomes (TEXE, TEGR and PROS). 

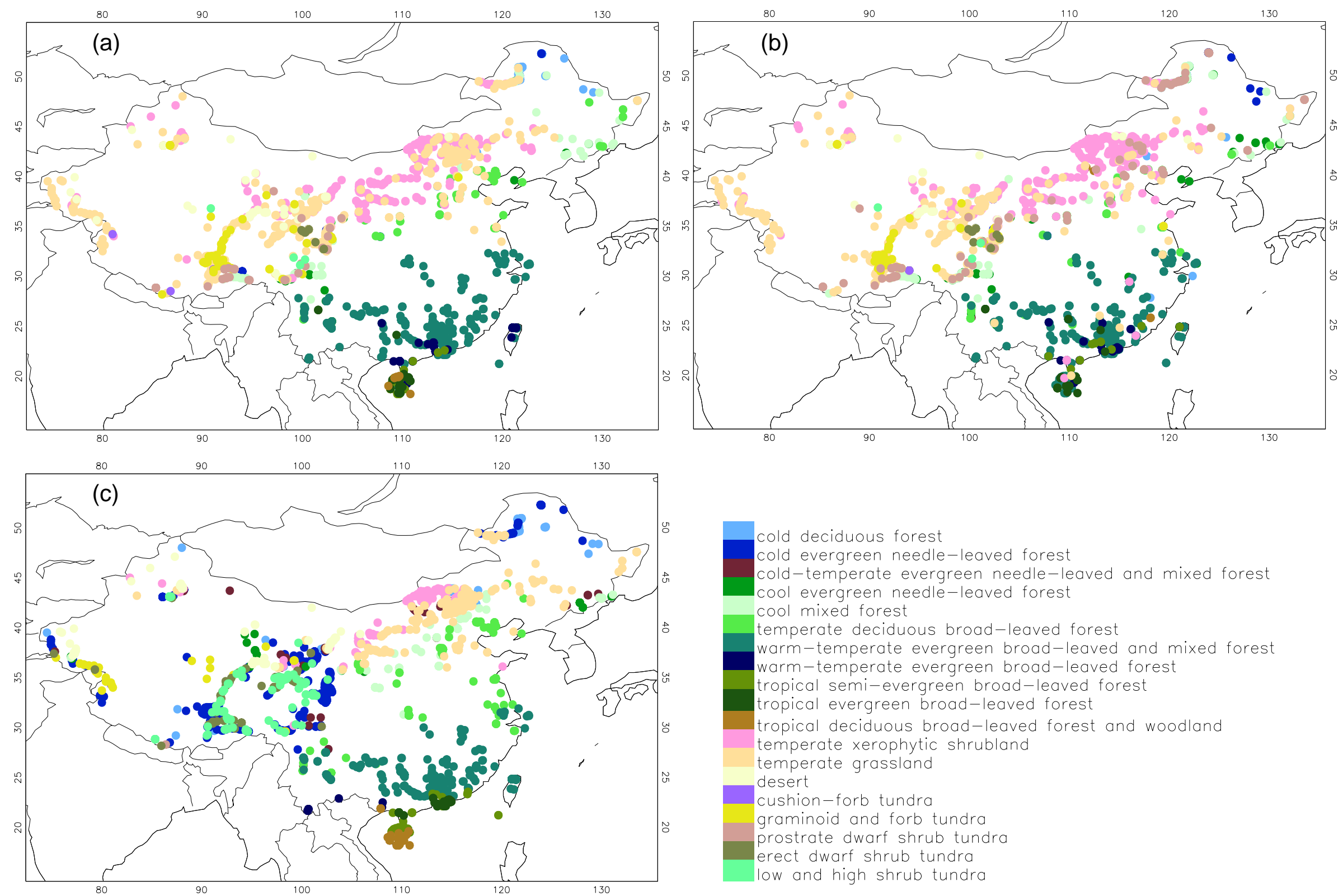

deciduous forest

cold evergreen needle-leaved forest

cold-temperate evergreen needle-leaved and mixed forest cool evergreen needle-leaved forest cool mixed forest

temperate deciduous broad-leaved forest

warm-temperate evergreen broad-leaved and mixed forest warm-temperate evergreen broad-leaved forest

tropical semi-evergreen broad-leaved forest

tropical evergreen broad-leaved forest

tropical deciduous broad-leaved forest and woodland temperate xerophytic shrubland temperate grassland desert

cushion-forb tundra

graminoid and forb tundra

prostrate dwarf shrub tundra

erect dwarf shrub tundra

Fig. 2. The observed modern biomes (a) based on field observations and vegetation map of China, the reconstructed biomes (b) based on modern surface pollen, and the potential biome (c) predicted by BIOME4 model. 


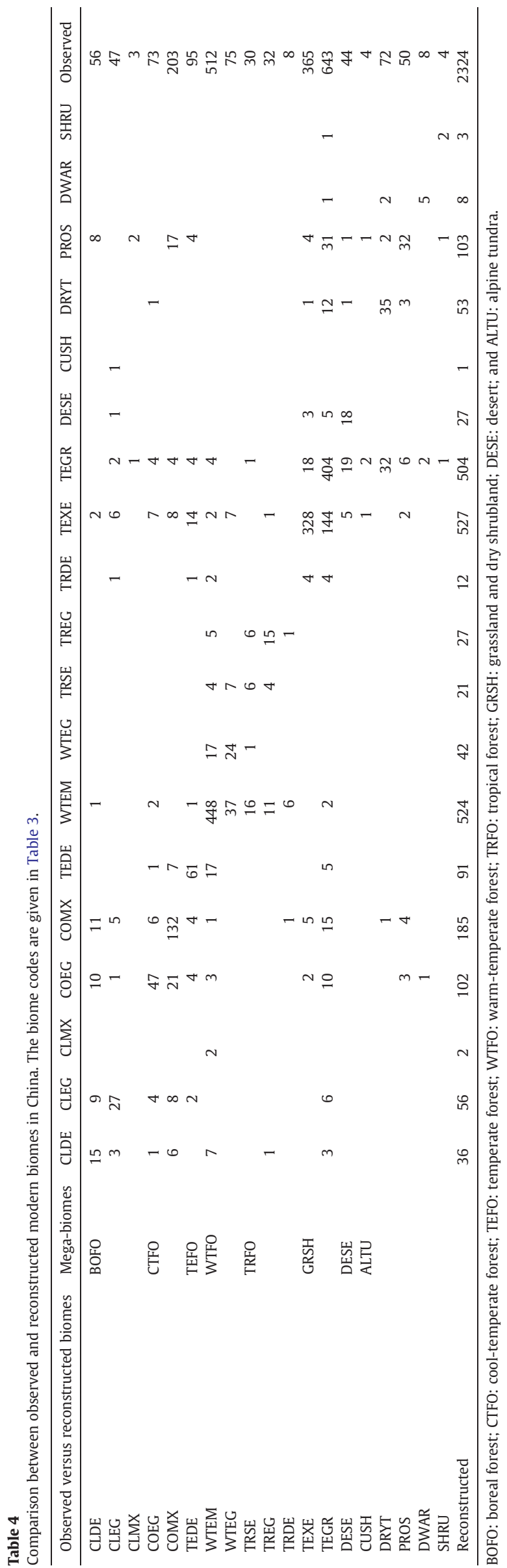

The incorrect assignment of 17 WTEM samples leads to a slight southern expansion of this forest (Fig. 2b).

WTEM and the pure warm-temperate evergreen broad-leaved forest (WTEG) are mainly distributed in the wide subtropical regions of southern China (Table 2). Compared to other forest biomes, the reconstruction of WTEM was the most accurate (87.5\%) which fits well with our field observations (Fig. 2a and b). However there remain 17 sites which were incorrectly assigned to WTEG and a further 17 sites which were assigned to TEDE whilst some other sites were assigned to forest and non-forest biomes (Table 4). The WTEG biome is mostly distributed in areas between WTEM and tropical vegetation, but also often occurs in the WTEM area (Fig. 2a and Table 2). In comparison, WTEG was less well reconstructed (32\%) than WTEM and it has been wrongly assigned to WTEM (Table 4) because they share the same evergreen woody PFTs (Table 3).

Tropical semi-evergreen broad-leaved forest (TRSE), tropical evergreen broad-leaved forest (TREG) and tropical deciduous broadleaved forest and woodland (TRDE) occur in the southernmost mainland of China, Hainan Island and Taiwan (Table 2). These biomes were not always correctly reconstructed, and were often confused with each other and with the warm-temperate forests (Table 4). There was no assignment of TRDE which was wrongly assigned to WTEM and from temperate shrubland and grassland (Table 4).

TEXE and TEGR coexist in the same arid and semi-arid areas of northern and northwestern China and on the Tibetan Plateau (Fig. 2a and Table 2). The pollen samples (accounting for $43 \%$ of the surface sites) were quite well reconstructed at $90 \%$ and $63 \%$ for TEXE and TEGR, respectively (Fig. 2b and Table 4), but they were often confused with each other and were also wrongly identified as cold-temperate forests, desert and tundra biomes, particularly the graminoid and forb tundra (DRYT) and PROS, and vice versa (Table 4).

The correctly reconstructed desert biome (DESE), occupying approximately $20 \%$ of the whole country and stretching from central northern to western China (ECVC, 1980), was found to be $40.9 \%$ (Fig. 2b) as some samples on the Tibetan Plateau were incorrectly assigned to the TEGR, TEXE and tundra biomes. Eight samples from TEXE and TEGR were also wrongly identified as DESE (Table 4).

Modern pollen samples assigned to tundra biomes are largely distributed on the Tibetan Plateau (Fig. 2a). Only four samples of cushion-forb tundra (CUSH) were reconstructed. Four other tundra biomes: DRYT, PROS, erect dwarf-shrub tundra (DWAR) and SHRU were all only partially reconstructed (Table 4). However DRYT was often confused with TEGR, whereas TEGR, CLDE and COMX were all wrongly identified as PROS (Table 4). The tundra biomes of DRYT and PROS occur in the Daxingan and Changbai Mountains and on the Inner Mongolian and Loess Plateaus (Fig. 2b) which should mainly consist of TEGR and cold/cool-temperate forests (Fig. 2a).

\subsubsection{Factors affecting biome assignment and reconstruction}

Map and data comparisons indicated that there is good agreement between the observed and reconstructed modern Chinese biomes, but incorrect assignments have occurred in some instances. Previous pollen-based biome reconstructions in China encountered similar problems. These include the incorrect assignment of cold- and cooltemperate forests, tropical and warm-temperate forests, and steppe, desert and tundra biomes (Yu et al., 1998, 2000; Ni et al., 2010). The same problems also exist in central and northern Eurasia (Tarasov et al., 1998) where tundra and forest biomes (taiga or cold deciduous forest) and desert and steppe biomes were mistaken for each other, especially in large river valleys and lakes. Taiga and cool conifer forests and cold and cool mixed forests and temperate deciduous forest were wrongly assigned in some places (Tarasov et al., 1998). These issues were also encountered in other European surface samples and are viewed as a weakness of the biomization method (Prentice et al., 1996). 

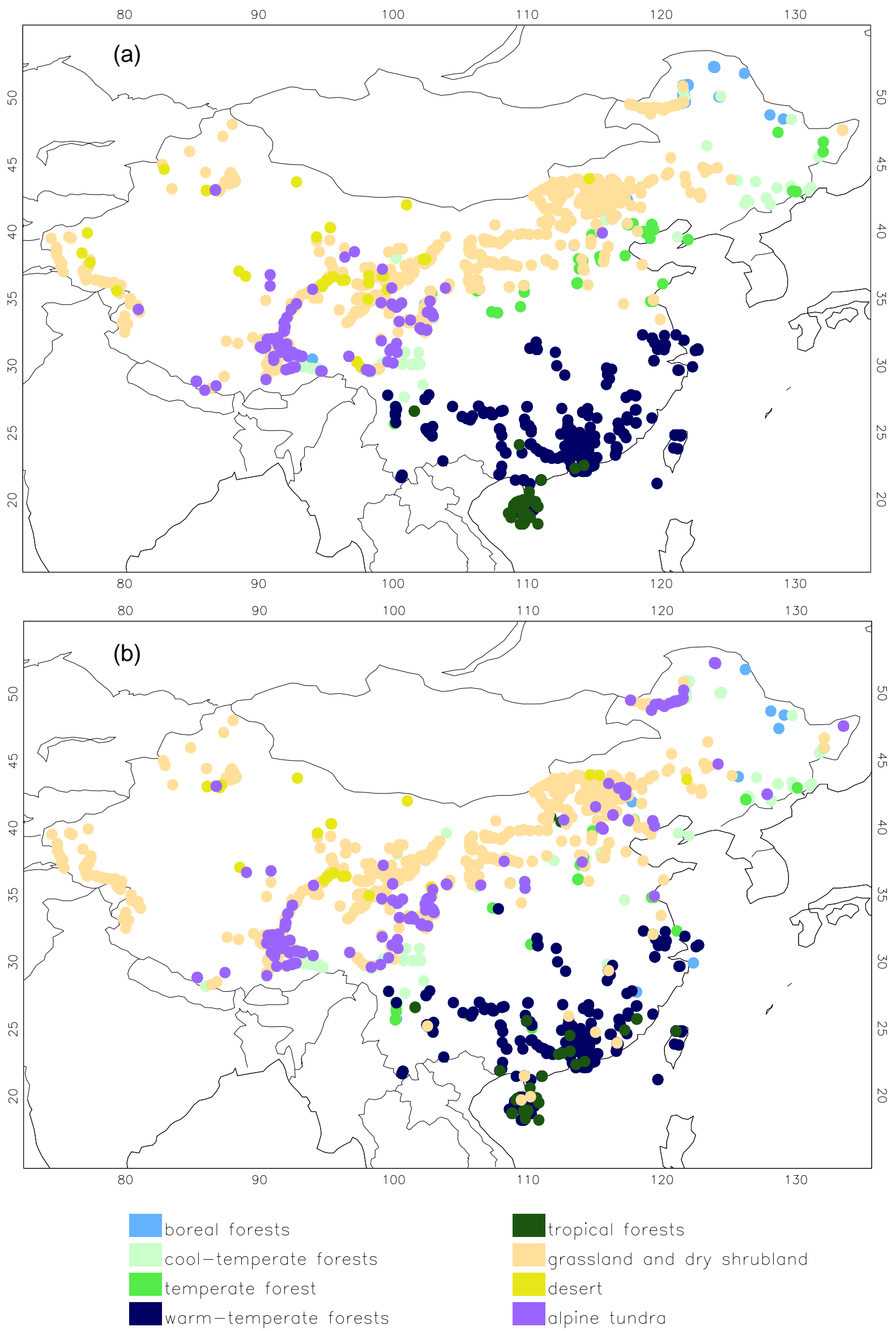

Fig. 3. Observed (a) and reconstructed (b) modern mega-biomes. 
There are several factors which account for the incorrect assignment of modern biomes in China. The principal reason is that biomes within the same or neighboring bioclimatic zones (e.g. coldtemperate and cool-temperate, and warm-temperate and tropical zones) are defined by the same combination of PFTs which are clearly not distinguishable for each biome during biomization. Another factor to consider is the long-distance transportation of pollen grains from forests to shrubland, steppe and desert, and vice versa, and from low to high altitudes. This was confirmed in previous reconstructions from China and Eurasia (Tarasov et al., 1998; Yu et al., 1998, 2000; Ni et al., 2010). In addition, pollen production, representation and dispersion affect the agreement between pollen and vegetation at local and regional scales (e.g. Prentice, 1985). Quantitative vegetation reconstruction based on pollen data should take these factors into account, e.g. by using model REVEALS and LOVE (Sugita, 2007a,b).

Pollen identification is also a key problem which is difficult to resolve. The assignment from pollen taxa to PFTs (and then to biomes) at family and genus levels rather than at species level reduced the amount of ecological information available for biome assignment. For example, the genus Pinus is found in various forest vegetation zones in eastern China and in mountainous regions. Pinus sylvestris var. mongolica is an indicator of cold mixed forest, whilst Pinus koraiensis is found in cool mixed forests, Pinus tabulaeformis in temperate deciduous forests, Pinus massoniana in warm-temperate forests, and Pinus yunnanensis is an indicator of tropical forest (ECVC, 1980). The difficulty in identifying these species within pollen records makes it almost impossible to distinguish biomes based on the Pinus genus.

It is also likely that anthropogenic modification of pollen assemblages has affected the assignment of pollen to biome, by the plantation of coniferous trees (e.g. Pinus and Cunninghamia) in many forest areas of eastern China, and agricultural activities across the whole country. The under-representation of some pollen taxa is an unresolved palynological problem for vegetation reconstruction. Problems with modern vegetation observation and mapping also influence the comparison of biomes.

\subsection{Biome reconstruction and simulation}

Comparing the observed (Fig. 2a) and reconstructed biomes (Fig. 2b) with the vegetation model-simulated biome data (Fig. 2c) indicates low correlations of $28.4 \%$ and $24.8 \%$, respectively (data not shown). Instances of incorrect identification occurred in all biomes, especially the cool-temperate forests, warm-temperate forests, temperate grassland and shrubland, and tundra biomes. This reveals a large discrepancy between the 'real' and potential vegetation.

The global vegetation models of the BIOME family have been successfully used in modern, past and future simulations of largescale vegetation (Prentice et al., 1992, 2007; Kaplan, 2001; Kaplan et al., 2003). BIOME3 does a good job of predicting the biomes in China (Ni et al., 2000). The BIOME4 model developed from the BIOME3 also does a reasonable job of simulating the pattern of Chinese biomes $(\mathrm{Ni}$, unpublished data). However the model simulation is performed on the basis of grid cells. The dominant biome for each grid cell of $10 \times 10 \mathrm{~km}^{2}$ was simulated, but as the pollen-based reconstruction is site-specific the larger grid cell area does not accurately reflect the dominant biome. This is one of the main reasons for the discrepancy between the reconstructed and simulated biomes. Vegetation models also have their shortcomings in simulating global and regional vegetation (e.g. Kaplan, 2001; Prentice et al., 2007). Vegetation simulation in China has similar problems (e.g. Ni et al., 2000) which impact upon comparisons between reconstructed and simulated biomes. Biases in climate and soil data furthermore reduce the accuracy of biome simulation. The considerable problems associated with biome assignment from vegetation types and the disadvantages of biomization described previously should be carefully considered prior to combining palaeo-environmental data with model outputs for modern pollen assemblages. The anthropogenic biome impact on biomes also needs to be assessed and involved in vegetation modeling and in the pollen biomization procedure.

\subsection{Mega-biome comparison}

Biomes which have similar bioclimatic controls and share the same PFTs were often confused with each other (Fig. 2a and b). This makes direct comparisons between biomes somewhat ambiguous. We have therefore grouped biomes that occur within the same bioclimatic zone into mega-biomes. This reclassification results in a more accurate spatial pattern between biomes (Fig. 3). Boreal forest (BOFO) occupied the coldest northeastern and northwestern areas of China and southeast Tibet (Fig. 3a; some sites in the northwest and Tibet were concealed by the altitudinal distribution of other sites), was correctly allocated in more than 50\% of places (Fig. 3b and Table 5). Cool-temperate forest (CTFO) was correctly reconstructed in northern China and on the southeast Tibetan Plateau (75\%). Temperate forest (TEFO) was distributed across the central and northern regions of China, whilst the warm-temperate forest mega-biome (WTFO) was correctly assigned in $90 \%$ of areas and occurs in subtropical southern China (Fig. 3 and Table 5). However tropical forest (TRFO) distributed in the southernmost Chinese mainland and in Taiwan and Hainan (Fig. 3a and b) was only correctly assigned in 50\% of cases. The grassland and dry shrubland mega-biome (GRSH) in northern and western China (Fig. 3a) has a correlation factor of $89 \%$ (Table 5). However this value decreases in subtropical China (Fig. 3b) as a result of anthropogenic activity. The desert biome remains the same as previously described, but a poor correlation is clear between GRSH and tundra (Table 5). All tundra biomes (CUSH, DRYT, PROS DWAR and SHRU) were grouped into one mega-biome of alpine tundra (ALTU). Its modern distribution occurs mostly on the Tibetan Plateau and in the high mountains of northern and western China (Fig. 3a), however there were also a number of incorrect assignments in the eastern part of northern China (Fig. 3b) with a correlation of $60 \%$ (Table 5). Overall, the regrouping of the observed and reconstructed biomes (Fig. 3) shows greater agreement (80.6\%) than the nongrouped biomes (Fig. 2).

Table 5

Comparison between observed and reconstructed modern mega-biomes in China.

\begin{tabular}{|c|c|c|c|c|c|c|c|c|c|}
\hline Observed versus reconstructed biomes & BOFO & CTFO & TEFO & WTFO & TRFO & GRSH & DESE & ALTU & Observed \\
\hline Boreal forest (BOFO) & 54 & 27 & & 1 & 1 & 11 & 1 & 11 & 106 \\
\hline Cool-temperate forest (CTFO) & 19 & 206 & 8 & 2 & & 23 & & 18 & 276 \\
\hline Temperate forest (TEFO) & 2 & 8 & 61 & 1 & 1 & 18 & & 4 & 95 \\
\hline Warm-temperate forest (WTFO) & 9 & 4 & 17 & 526 & 18 & 13 & & & 587 \\
\hline Tropical forest (TRFO) & 1 & 1 & & 34 & 32 & 2 & & & 70 \\
\hline Grassland and dry shrubland (GRSH) & 9 & 32 & 5 & 2 & 8 & 894 & 8 & 50 & 1008 \\
\hline Desert (DESE) & & & & & & 24 & 18 & 2 & 44 \\
\hline Alpine tundra (ALTU) & & 9 & & & & 46 & & 83 & 138 \\
\hline Reconstructed & 94 & 287 & 91 & 566 & 60 & 1031 & 27 & 168 & 2324 \\
\hline
\end{tabular}




\subsection{Altitudinal biome distribution}

Whilst all of the previously described biomes and mega-biomes have marked zonal distribution changes, altitudinal changes are often not clearly highlighted (Figs. 2 and 3 ). Therefore the biome distributions within six mountainous regions were also separately mapped (Fig. 4). In the Daxingan Mountains of northeast China, the vertical distribution of observed biomes from lower to higher elevations is TEXE, TEGR, COMX and then CLDE (Fig. 4a). A number of samples of TEGR were wrongly assigned as PROS and some of CLDE were wrongly assigned as COEG and COMX. There are overlaps between the different biome boundaries and also altitudinal biome change is not obvious because of the relatively low altitudes (Fig. 4a).

The Tianshan Mountains in northwestern China has different vegetation patterns on the northern (alpine and subalpine meadow, montane conifer forest, steppe and shrubland, and desert) and

observed

reconstructed

observed reconstructed

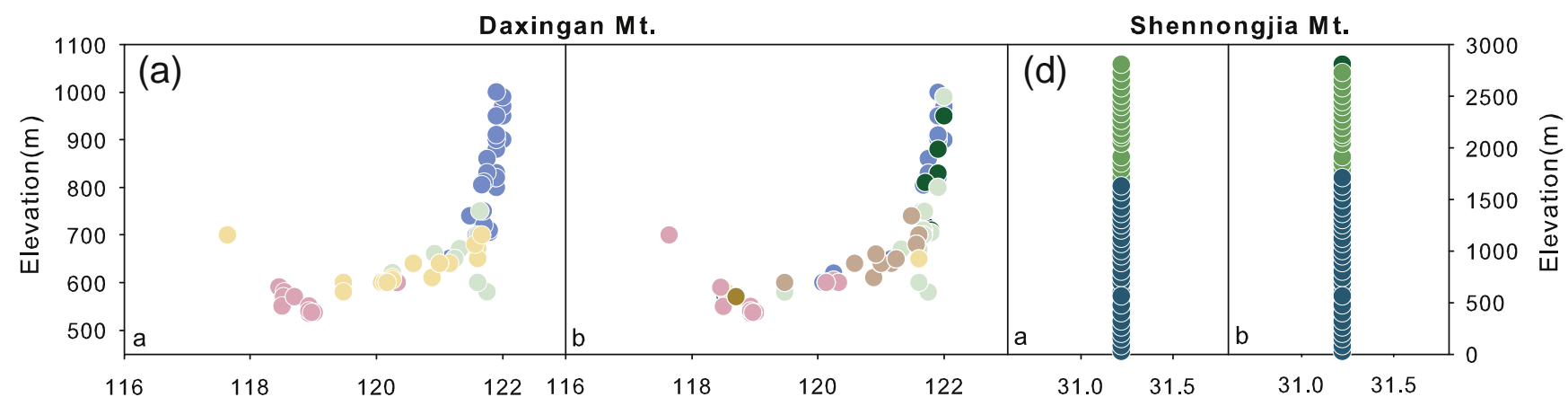

Tianshan $\mathbf{M t}$.
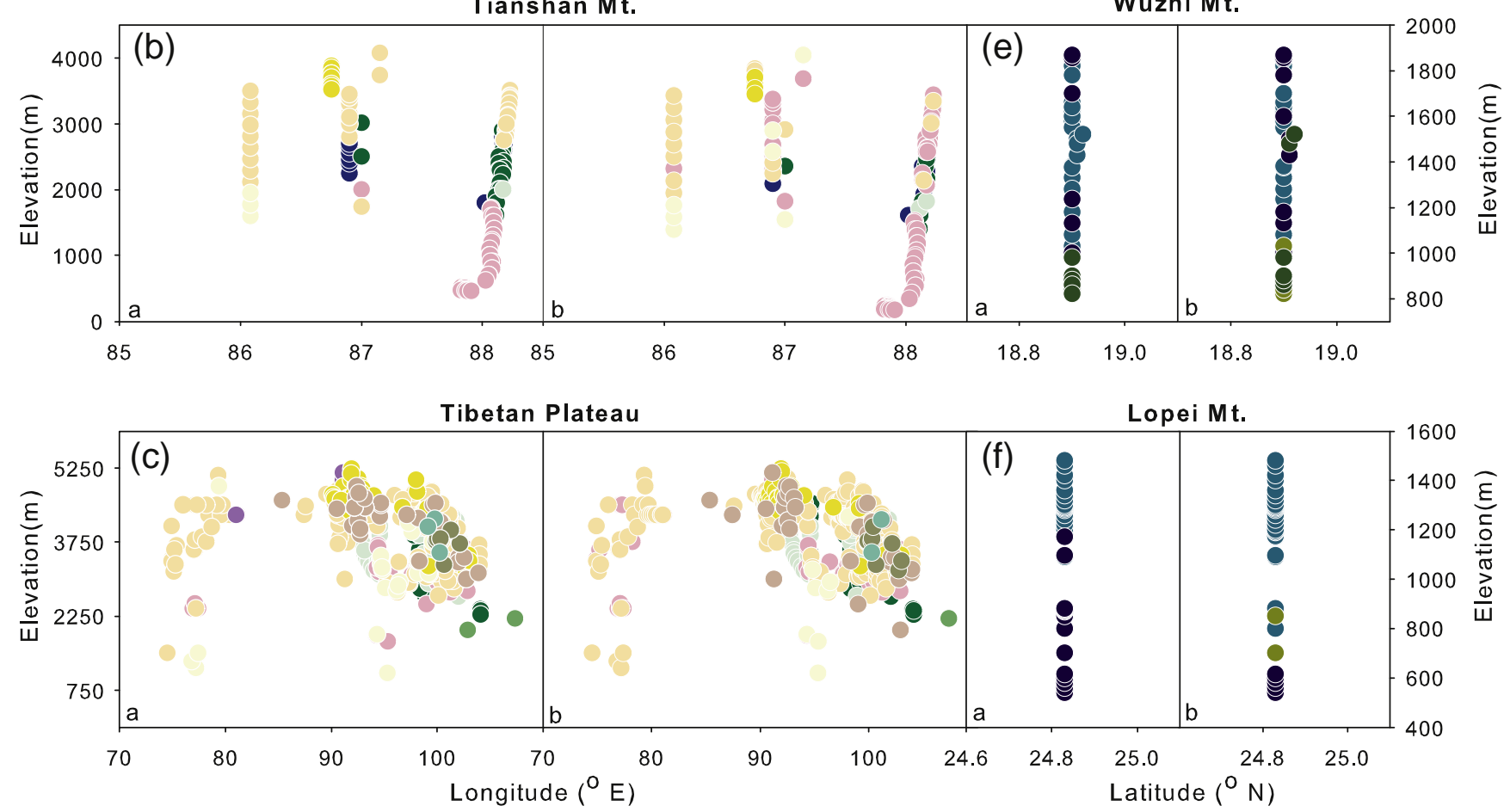

- cold deciduous forest

- cold evergreen needle-leaved forest

- cold-temperate evergreen needle-leaved and mixed forest

- cool evergreen needle-leaved forest

cool mixed forest

- temperate deciduous broad-leaved forest

- warm-temperate evergreen broad-leaved and mixed forest

- warm-temperate evergreen broad-leaved forest

- tropical semi-evergreen broad-leaved forest

- tropical evergreen broad-leaved forest
- tropical deciduous broad-leaved forest and woodland

- temperate xerophytic shrubland

temperate grassland

desert

- cushion-forb tundra

- graminoid and forb tundra

- prostrate dwarf shrub tundra

- erect dwarf shrub tundra

- low and high shrub tundra

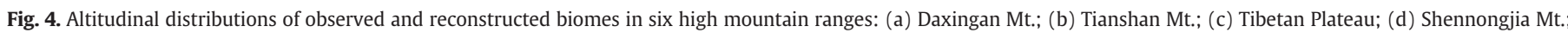
(e) Wuzhi Mt.; and (f) Lopei Mt. 
southern slopes (steppe and sparse succulent vegetation). Pollen data from the northern slopes indicate a gradient change between several biomes: shrubland and desert in the low elevations, needle-leaved forests at mid-elevations, and steppe (meadow) mixed with small amounts of tundra at higher elevations. The reconstructed TEXE biome covers a large altitudinal range in the Tianshan Mountains (Fig. 4b). An incorrect assignment of xerophytic shrubland at high elevations is due to the transport of shrub pollen from the lowland deserts to the upland regions.

The Tibetan Plateau has extremely complex vegetation patterns as shown in Fig. 2. There is a general trend from subalpine coniferous forest and shrubland in the southeast, to alpine meadow and alpine steppe in the central region, and to alpine desert in the northwest (ECVC, 1980). A total of 616 samples from the Tibetan Plateau produced 11 biomes, which show a good gradient change that is comparable with our observations (Fig. 4c).

The reconstructed biomes for the northern subtropical mountains of Shennongjia are also in good agreement with our observations: WTEM occurs at low elevations and TEDE at higher elevations (Fig. 4d). There are no high mountains in tropical China, however the mid-high mountain ranges of Wuzhi on Hainan Island contain TRSE and TREG at elevations of $<1000 \mathrm{~m}$, WTEG between 1000 and $1900 \mathrm{~m}$, and WTEM between 1800 and $2000 \mathrm{~m}$ (Fig. 4e). Three TREG sites were incorrectly identified as WTEM, whilst there was confusion between WTEG and WTEM at several sites (Fig. 4e). In the Lopei Mountains of Taiwan the reconstructed biomes show that WTEG occurs below $1200 \mathrm{~m}$ and WTEM above $1200 \mathrm{~m}$ except where some of the WTEG samples have been wrongly identified as TRSE and WTEM (Fig. 4f).

\section{Conclusions and perspectives}

(1) The surface pollen data set is more extensive, both in terms of geographical coverage and pollen samples and taxa included, than previous data sets used in the biomization (Yu et al., 1998, 2000; Ni et al., 2010) thus making our modern pollen-based biome reconstruction more robust.

Whilst 554 digitized pollen samples have incomplete pollen assemblages (reduced or combined) and less accurate pollen records, this has not affected the final pollen-biome assignments because all digitized samples included key pollen taxa that characterize the biomes through the assignment of PFTs. In addition, there are still geographical gaps in the collection of surface pollen samples, for example in the central and northwestern Tibetan Plateau, the desert regions of Tarim Basin in southern Xinjiang Autonomous Region, on Alashan Plateau in western Inner Mongolia, the mountainous regions of eastern China and parts of eastern China which have been highly affected by human activity (Fig. 1). In these regions there has either been no surface pollen sampling or no collection of existing samples. More extensive sample collections are therefore required in these areas. Complete pollen assemblages and records with raw data are always desirable.

(2) The globally tested, robust biomization technique has been improved in this study by the assignment of pollen-PFTs and the classification of biomes. This has made the modern pollenbased biome reconstruction more reliable.

The biomization method has been broadly tested for modern and past biome reconstructions on continental and regional scales (Prentice and Webb, 1998; Prentice et al., 2000). The newly established global PFT scheme has reasonable plant traits-based ecophysiological and bioclimatic foundations (Harrison et al., 2010), and has been successfully used in pollen-based reconstruction of present and past vegetation $(\mathrm{Ni}$ et al., 2010). The assignment of pollen taxa to PFTs has been updated and calibrated based on the recent availability of updated Chinese floras. Biomes have been defined in detail based on regional vegetation information and can also be compared to global biome classifications. Our biome reconstruction based on this updated biomization method is therefore more reliable.

We adopted the standard biomization technique, i.e., only assignments from pollen taxa to PFTs and from PFTs to biomes were used and other rules in the biomization procedure (Prentice et al., 1996; Prentice and Webb, 1998) were not changed. Regional alterations such as pollen percentage thresholds and weighting for some key taxa should be taken into account in further research.

(3) Multiple comparisons between biome reconstructions, observed biomes, observed potential biomes, and biome simulations provide insights into quantitative modern pollen-vegetation relationships. This benefits past vegetation simulations and efforts to combine modelling and palaeo-environmental data as well as multi-proxy biome reconstructions.

The reconstructed biomes were compared with modern vegetation information obtained from direct field observations, the natural and agricultural vegetation patterns from digitized atlases, and the vegetation model-simulated biomes. Whilst there is good correlation between the biome reconstruction and biomes derived from other sources, there is a low correlation rate between reconstructed and simulated biomes. This will hopefully lead to new research comparing pollen data with models in the future resulting in more realistic past vegetation modeling.

(4) The human impact on modern surface pollen assemblages was only considered in parts of this study. Whilst not considered in this study, the large-scale reconstruction of anthropogenic biomes based on modern pollen records presents a challenge for future pollen research.

More than $75 \%$ of the ice-free land on Earth has been altered by human activity, which has modified global patterns of biodiversity and ecosystem processes (Ellis and Ramankutty, 2008). The anthropogenic biome has therefore become an important research target for Earth System Science, especially given current global change (Alessa and Chapin, 2008). The study of regionalscale climate-human-environmental interactions is essential for fully understanding global environmental changes, both at present and in the past and future, and anthropogenically impacted large-scale pollen studies could play a key role.

However we must pay more attention to the careful treatment of human-disturbed surface pollen samples when apply them to reconstruct biomes and past climates. Some of highly modified samples that might result in misunderstanding and confusion of local vegetation and climate may be excluded from pollen dataset.

Supplementary data associated with this article can be found, in the online version, at doi:10.1016/j.gloplacha.2010.09.002.

\section{Acknowledgements}

This study was supported by the German Research Foundation (DFG SPP 1266, He 3622/11) and the National Natural Science Foundation of China (NSFC 30590383). The pollen data collection and all of the early studies were supported through various grants from the NSFC (39700018, 90102009), the Chinese Academy of Sciences (CAS KZCX1-10-05), and the Institute of Botany CAS. We thank many palynologists in China, especially Zhaochen Kong, Qinghai Xu, Shun Yan, Ge Yu and Zhuo Zheng for providing surface pollen data. We are also grateful to Tingting Huo for digitizing some of pollen diagrams, to Han Wang for extracting vegetation maps from the vegetation atlas of China, and to Katy Wilson for improving the English language of this manuscript. We deeply appreciate three reviewers for their very 
helpful comments and suggestions which help us greatly improve the manuscript.

\section{References}

Alessa, L., Chapin III, F.S., 2008. Anthropogenic biomes: a key contribution to earthsystem science. Trends in Ecology and Evolution 23, 529-531.

Anderson, P.M., Bartlein, P.J., Brubaker, L.B., Gajewski, K., Ritchie, J.C., 1991. Vegetation pollen climate relationships for the arcto-boreal region of North America and Greenland. Journal of Biogeography 18, 565-582.

Broström, A., Sugita, S., Gaillard, M.J., 2004. Pollen productivity estimates for the reconstruction of past vegetation cover in the cultural landscape of southern Sweden. Holocene 14, 368-381.

Cour, P., Zheng, Z., Duzer, D., Calleja, M., Yao, Z., 1999. Vegetational and climatic significance of modern pollen rain in northwestern Tibet. Review of Palaeobotany and Palynology 104, 183-204.

Court-Picon, M., Buttler, A., de Beaulieu, J.L., 2006. Modern pollen/vegetation/land-use relationships in mountain environments: an example from the Champsaur valley (French Alps). Vegetation History and Archaeobotany 15, 151-168.

EBVAC (Editorial Board of Vegetation Atlas of China, Chinese Academy of Sciences), 2001. Vegetation Atlas of China. Science Press, Beijing. In Chinese.

ECFC (Editorial Committee of Flora of China), 1959-2002. Flora of China (Chinese Edition). Science Press, Beijing.

ECVC (Editorial Committee for Vegetation of China), 1980. Vegetation of China. Science Press, Beijing. In Chinese.

Ellis, E.C., Ramankutty, N., 2008. Putting people on the map: anthropogenic biomes of the world. Frontiers in Ecology and the Environment 6, 439-447.

Farr, T.G., Rosen, P.A., Caro, E., Crippen, R., Duren, R., Hensley, S., Kobrick, M., Paller, M., Rodriguez, E., Roth, L., Seal, D., Shaffer, S., Shimada, J., Umland, J., Werner, M., Oskin, M., Burbank, D., Alsdorf, D., 2007. The shuttle radar topography mission. Reviews of Geophysics 45, Art. No. RG2004. doi:10.1029/2005RG000183.

Finsinger, W., Heiri, O., Valsecchi, V., Tinner, W., Lotter, A.F., 2007. Modern pollen assemblages as climate indicators in southern Europe. Global Ecology and Biogeography 16, 567-582.

Fu, L.G., Chen, T.Q., Lang, K.Y., Hong, T., Lin, Q., Li, Y., 1999-2009. High Plants of China. Qingdao Publishing House, Qingdao.

Gaillard, M.J., Birks, H.J.B., Emanuelsson, U., Karlsson, S., Lageras, P., Olausson, D., 1994 Application of modern pollen land-use relationships to the interpretation of pollen diagrams - reconstructions of land-use history in South Sweden, 3000-0 BP. Review of Palaeobotany and Palynology 82, 47-73.

Gaillard, M.J., Sugita, S., Bunting, J., Dearing, J., Bittmann, F., 2008. Human impact on terrestrial ecosystems, pollen calibration and quantitative reconstruction of past land-cover. Vegetation History and Archaeobotany 17, 415-418.

Gajewski, K., Lezine, A.M., Vincens, A., Delestan, A., Sawada, M., 2002. Modern climatevegetation-pollen relations in Africa and adjacent areas. Quaternary Science Reviews 21, 1611-1631.

Gotanda, K., Nakagawa, T., Tarasov, P.E., Yasuda, Y., 2008. Disturbed vegetation reconstruction using the biomization method from Japanese pollen data: Modern and Late Quaternary samples. Quaternary International 184, 56-74.

Harrison, S.P., Prentice, I.C., Barboni, D., Kohfeld, K., Ni, J., Sutra, J-P., in preparation. Towards a global plant functional type classification for ecosystem modelling, palaeoecology and climate impacts research.

Harrison, S.P., Prentice, I.C., Barboni, D., Kohfeld, K., Ni, J., Sutra, J.-P., 2010. Ecophysiological and bioclimatic foundations for a global plant functional classification. Journal of Vegetation Science 21, 300-317.

Herzschuh, U., 2007. Reliability of pollen ratios for environmental reconstructions on the Tibetan Plateau. Journal of Biogeography 34, 1265-1273.

Hjelle, K.L., 1999. Modern pollen assemblages from mown and grazed vegetation types in western Norway. Review of Palaeobotany and Palynology 107, 55-81.

Hou, K.Z., 1998. A Dictionary of the Families and Genera of Chinese Seed Plants, 2nd Edition. Science Press, Beijing. In Chinese.

Hutchinson, M.F., 2006. ANUSPLIN Version 4.36 User Guide. Centre for Resource and Environmental Studies, the Australian National University, Canberra.

Kaplan, J.O., 2001. Geophysical applications of vegetation modeling. Ph.D. Dissertation, Lund University, Lund.

Kaplan, J.O., Bigelow, N.H., Prentice, I.C., Harrison, S.P., Bartlein, P.J., Christensen, T.R Cramer, W., Matveyeve, N.V., McGuire, A.D., Murray, D.F., Razzhivin, V.Y., Smith, B., Walker, D.A., Anderson, P.M., Andreev, A.A., Brubaker, L.B., Edwards, M.E., Lozhkin, A.V., 2003. Climate change and Arctic ecosystems: 2. Modeling, paleodata-model comparisons, and future projections. Journal of Geophysical Research 108, 8171. doi:10.1029/2002JD002559.

Lebamba, J., Ngomanda, A., Vincens, A., Jolly, D., Favier, C., Elenga, H., Bentaleb, I., 2009. Central African biomes and forest succession stages derived from modern pollen data and plant functional types. Climate of the Past 5, 403-429.

Li, Y.C., Xu, Q.H., Yang, X.L., Chen, H., Lu, X.M., 2005. Pollen-vegetation relationship and pollen preservation on the Northeastern Qinghai-Tibetan Plateau. Grana 44, $160-171$.

Li, Y.C., Xu, Q.G., Liu, J.S., Yang, X.L., Nakagawa, T., 2007. A transfer-function model developed from an extensive surface-pollen data set in northern China and its potential for palaeoclimate reconstructions. Holocene 17, 897-905.

Liu, J.G., Diamond, J., 2005. China's environment in a globalizing world. Nature 435 1179-1186.

Liu, H.Y., Wang, Y., Tian, Y.H., Zhu, J.L., Wang, H.Y., 2006. Climatic and anthropogenic control of surface pollen assemblages in East Asian steppes. Review of Palaeobotany and Palynology 138, 281-289.
Liu, H.Y., Wei, F.L., Liu, K., Zhu, J.L., Wang, H.Y., 2008. Determinants of pollen dispersal in the East Asian steppe at different spatial scales. Review of Palaeobotany and Palynology 149, 219-228.

Lu, H.Y., Wu, N.Q., Yang, X.D., Shen, C.M., Zhu, L.P., Wang, L., Li, Q.A., Xu, D.K., Tong, G.B. Sun, X.J., 2008. Spatial pattern of Abies and Picea surface pollen distribution along the elevation gradient in the Qinghai-Tibetan Plateau and Xinjiang, China. Boreas 37, 254-262.

Ma, Y.Z., Liu, K.-B., Feng, Z.D., Sang, Y.L., Wang, W., Sun, A.Z., 2008. A survey of modern pollen and vegetation along a south-north transect in Mongolia. Journal of Biogeography 35, 1512-1532.

Marchant, R., Cleef, A., Harrison, S.P., Hooghiemstra, H., Markgraf, V., van Boxel, J., Ager, T., Almeida, L., Anderson, R., Baied, C., Behling, H., Berrio, J.C., Burbridge, R., Bjorck S., Byrne, R., Bush, M., Duivenvoorden, J., Flenley, J., De Oliveira, P., van Geel, B., Graf, K., Gosling, W.D., Harbele, S., van der Hammen, T., Hansen, B., Horn, S., Kuhry, P., Ledru, M.-P., Mayle, F., Leyden, B., Lozano-Garcia, S., Melief, A.M., Moreno, P., Moar, N.T., Prieto, A., van Reenen, G., Salgado-Labouriau, M., Schaebitz, F., SchreveBrinkman, E.J., Wille, M., 2009. Pollen-based biome reconstructions for Latin America at 0,6000 and 18000 radiocarbon years ago. Climate of the Past 5, 725-767.

Markgraf, V., Webb, R.S., Anderson, K.H., Anderson, L., 2002. Modern pollen/climate calibration for southern South America. Palaeogeography Palaeoclimatology Palaeoecology 181, 375-397.

Minckley, T.A., Bartlein, P.J., Whitlock, C., Shuman, B.N., Williams, J.W., Davis, O.K., 2008 Associations among modern pollen, vegetation, and climate in western North America. Quaternary Science Reviews 27, 1962-1991.

Newsome, J.C., 1999. Pollen-vegetation relationships in semi-arid southwestern Australia. Review of Palaeobotany and Palynology 106, 103-119.

Ni, J., Sykes, M.T., Prentice, I.C., Cramer, W., 2000. Modeling the vegetation of China using the process-based equilibrium terrestrial biosphere model BIOME3. Global Ecology and Biogeography 9, 463-479.

Ni, J., Yu, G., Harrison, S.P., Prentice, I.C., 2010. Palaeovegetation in China during the late Quaternary: biome reconstructions based on a global scheme of plant functional types. Palaeogeography, Palaeoclimatology, Palaeoecology 289, 44-61.

Overpeck, J.T., Webb, T., Prentice, I.C., 1985. Quantitative interpretation of fossil pollen spectra - dissimilarity coefficients and the method of modern analogs. Quaternary Research 23, 87-108.

Pickett, E.J., Harrison, S.P., Hope, G., Harle, K., Dodson, J.R., Kershaw, A.P., Prentice, I.C., Backhouse, J., Colhoun, E.A., D'Costa, D., Flenley, J., Grinddrod, J., Haberle, S., Hassell, C., Kenyon, C., Macphail, M., Martin, H., Martin, A.H., Mckenzie, M., Newsome, J.C. Penny, D., Powell, J., Raine, J.I., Southern, W., Stevenson, J., Sutra, J.P., Thomas, I., van der Kaars, S., Ward, J., 2004. Pollen-based reconstructions of biome distributions for Australia, Southeast Asia and the Pacific (SEAPAC region) at 0,6000 and $18,000{ }^{14} \mathrm{C}$ yr B.P. Journal of Biogeography 31, 1381-1444.

Prentice, I.C., 1985. Pollen representation, source area, and basin size - toward a unified theory of pollen analysis. Quaternary Research 23, 76-86.

Prentice, I.C., Webb, T., 1998. BIOME 6000: reconstructing global mid-Holocene vegetation patterns from palaeoecological records. Journal of Biogeography 25 , 997-1005.

Prentice, I.C., Cramer, W., Harrison, S.P., Leemans, R., Monserud, R.A., Solomon, A.M. 1992. A global biome model based on plant physiology and dominance, soil properties and climate. Journal of Biogeography 19, 117-134.

Prentice, I.C., Guiot, J., Huntley, B., Jolly, D., Cheddadi, R., 1996. Reconstructing biomes from palaeoecological data: a general method and its application to European pollen data at 0 and $6 \mathrm{ka}$. Climate Dynamics 12, 185-194.

Prentice, I.C., Jolly, D., BIOME 6000 participants, 2000. Mid-Holocene and glacialmaximum vegetation geography of the northern continents and Africa. Journal of Biogeography 27, 507-519.

Prentice, I.C., Bondeau, A., Cramer, W., Harrison, S.P., Hickler, T., Lucht, W., Sitch, S. Smith, B., Sykes, M.T., 2007. Dynamic global vegetation modeling: quantifying terrestrial ecosystem responses to large-scale environmental change. In: Canadell, J., Pitelka, L.F., Pataki, D. (Eds.), Terrestrial Ecosystems in a Changing World. Springer-Verlag, Berlin, Heidelberg, pp. 175-192.

Seppä, H., Birks, H.J.B., Odland, A., Poska, A., Veski, S., 2004. A modern pollen-climate calibration set from northern Europe: developing and testing a tool for palaeoclimatological reconstructions. Journal of Biogeography 31, 251-267.

Shen, C.M., Liu, K.B., Tang, L.Y., Overpeck, J.T., 2006. Quantitative relationships between modem pollen rain and climate in the Tibetan Plateau. Review of Palaeobotany and Palynology 140, 61-77.

Shen, C.M., Liu, K.B., Tang, L.Y., Overpeck, J.T., 2008. Numerical analysis of modern and fossil pollen data from the Tibetan Plateau. Annals of the Association of American Geographers 98, 755-772.

Sugita, S., 2007a. Theory of quantitative reconstruction of vegetation I: pollen from large sites REVEALS regional vegetation composition. The Holocene 17, 229-241.

Sugita, S., 2007b. Theory of quantitative reconstruction of vegetation II: all you need is LOVE. The Holocene 17, 243-257.

Tarasov, P.E., Webb, T., Andreev, A.A., Afanas'eva, N.B., Berezina, N.A., Bezusko, L.G., Blyakharchuk, T.A., Bolikhovskaya, N.S., Cheddadi, R., Chernavskaya, M.M. Chernova, G.M., Dorofeyuk, N.I., Dirksen, V.G., Elina, G.A., Filimonova, L.V., Glebov, F.Z., Guiot, J., Gunova, V.S., Harrison, S.P., Jolly, D., Khomutova, V.I., Kvavadze, E.V., Osipova, I.M., Panova, N.K., Prentice, I.C., Saarse, L., Sevastyanov, D.V., Volkova, V.S. Zernitskaya, V.P., 1998. Present-day and mid-Holocene biomes reconstructed from pollen and plant macrofossil data from the former Soviet Union and Mongolia. Journal of Biogeography 25, 1029-1053.

Vincens, A., Bremond, L., Brewer, S., Buchet, G., Dussouillez, P., 2006. Modern pollenbased biome reconstructions in East Africa expanded to southern Tanzania. Review of Palaeobotany and Palynology 140, 187-212. 
Watrin, J., Lezine, A.M., Gajewski, K., Vincens, A., 2007. Pollen-plant-climate relationships in sub-Saharan Africa. Journal of Biogeography 34, 489-499.

Whitmore, J., Gajewski, K., Sawada, M., Williams, J.W., Shuman, B., Bartlein, P.J., Minckley, T., Viau, A.E., Webb, T., Shafer, S., Anderson, P., Brubaker, L., 2005. Modern pollen data from North American and Greenland for multi-scale paleoenvironmental applications. Quaternary Science Reviews 24, 1828-1848.

Wu, Z.H., Zhu, J.N., Yang, C.Y., 1992. A Dictionary of the Extant and Fossil Families and Genera of Chinese Ferns. China Science and Technology Press, Beijing.

Wu, Z.Y., Raven, P.H., Hong, D.Y., 1994-2009. Flora of China. Science Press and Missouri Botanical Garden Press (St. Louis, Beijing and Missouri.

Xu, Q.H., Li, Y.C., Yang, X.L., Zheng, Z.H., 2007. Quantitative relationship between pollen and vegetation in northern China. Science in China Series D-Earth Sciences 50, 582-599.

Xu, Q.H., Li, Y.C., Tian, F., Cao, X.Y., Yang, X.L., 2009. Pollen assemblages of tauber traps and surface soil samples in steppe areas of China and their relationships with vegetation and climate. Review of Palaeobotany and Palynology 153, 86-101.

Yu, G., Prentice, I.C., Harrison, S.P., Sun, X.J., 1998. Pollen-based biome reconstructions for China at 0 and 6000 years. Journal of Biogeography 25, 1055-1069.
Yu, G., Chen, X., Ni, J., Cheddadi, R., Guiot, J., Han, H., Harrison, S.P., Huang, C., He, M., Kong, Z., Li, S., Li, W., Liew, P., Liu, G., Liu, J., Liu, K.-B., Prentice, I.C., Qui, W., Ren, G., Song, C., Sugita, S., Sun, X., Tang, L., Van Campo, E., Xia, Y., Xu, Q., Yan, S., Yang, X., Zhao, J., Zheng, Z., 2000. Palaeovegetation of China: a pollen data-based synthesis for the mid-Holocene and last glacial maximum. Journal of Biogeography 27, 635-664.

Yu, G., Tang, L.Y., Yang, X.D., Ke, X.K., Harrison, S.P., 2001. Modern pollen samples from alpine vegetation on the Tibetan Plateau. Global Ecology and Biogeography 10, 503-520.

Yu, G., Kee, X.K., Xue, B., Ni, J., 2004. The relationships between the surface arboreal pollen and the plants of the vegetation in China. Review of Palaeobotany and Palynology 129, 187-198.

Zheng, Z., Cour, P., Huang, C.X., Duzer, D., Robert, G., Calleja, M., Beaudouin, C., Deng, Y., Huang, K.Y., 2007. Dust pollen distribution on a continental scale and its relation to present-day vegetation along north-south transects in east China. Science in China Series D: Earth Sciences 50, 236-246. 